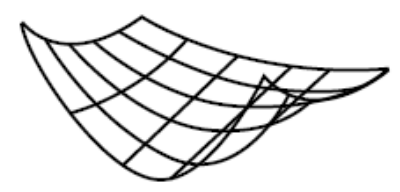

UNIVERSITY OF

TECHNOLOGY SYDNEY

QUANTITATIVE FINANCE

RESEARCH CENTRE

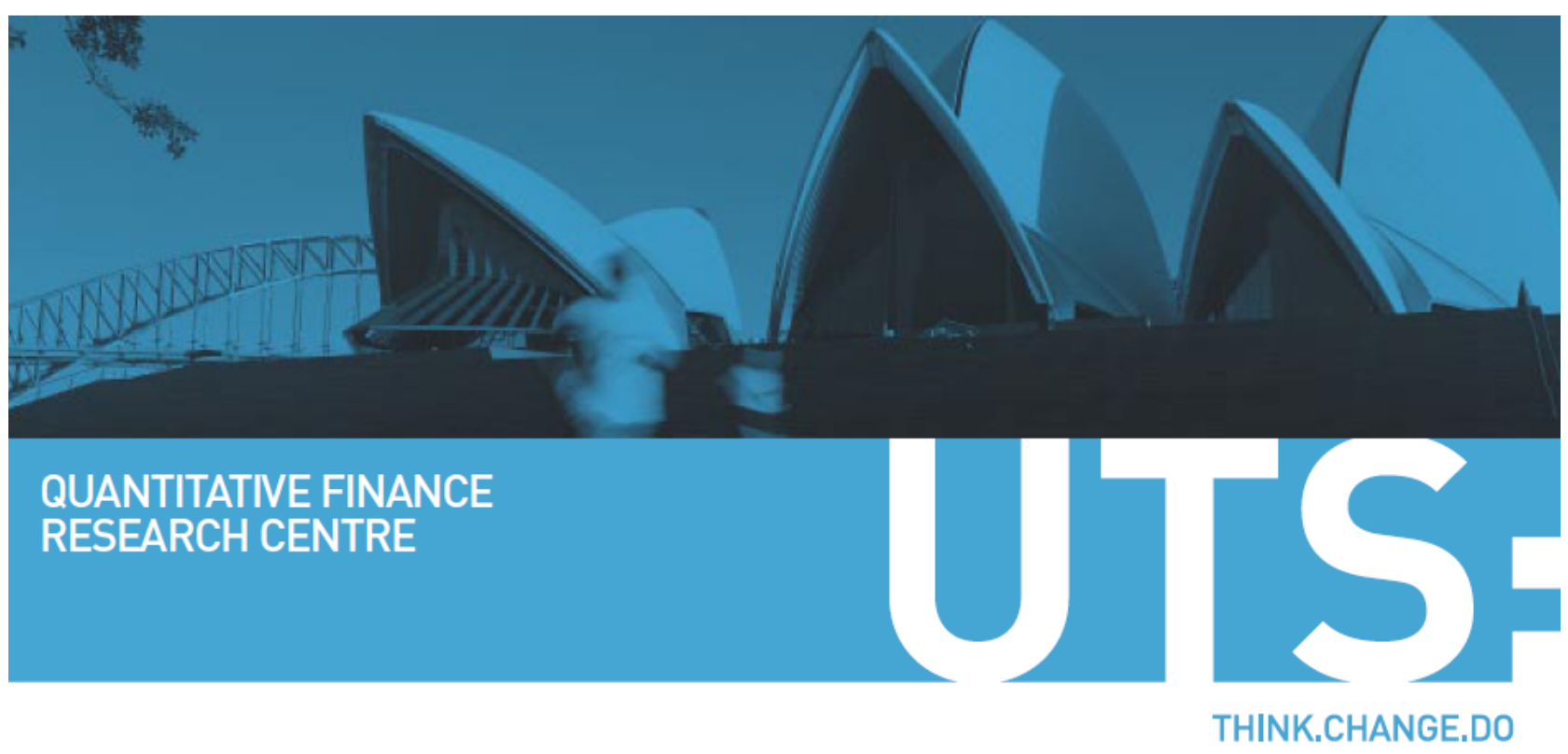

QUANTITATIVE FINANCE RESEARCH CENTRE

Equity-Linked Pension Schemes with Guarantees

J. Aase Nielsen, Klaus Sandmann and Erik Schlögl 


\title{
Equity-Linked Pension Schemes with Guarantees
}

\author{
J. Aase Nielsen ${ }^{1}$, Klaus Sandmann ${ }^{2}$ and Erik Schlögl ${ }^{3}$
}

\begin{abstract}
:
This paper analyses the relationship between the level of a return guarantee in an equity-linked pension scheme and the proportion of an investor's contribution needed to finance this guarantee. Three types of schemes are considered: investment guarantee, contribution guarantee and participation surplus. The evaluation of each scheme involves pricing an Asian option, for which relatively tight upper and lower bounds can be calculated in a numerically efficient manner.

We find a negative (and for two contract specifications also concave) relationship between the participation in the surplus return of the investment strategy and the guarantee level in terms of a minimum rate of return. Furthermore, the introduction of a possibility of early termination of the contract (e.g. due to the death of the investor) has no qualitative and very little quantitative impact on this relationship.
\end{abstract}

Version: December 2009

Key words: Pension funds, forward risk adjusted measure, Asian option.

JEL Classification: G13, G23

\section{Introduction}

Over the past decade, defined-contribution (as opposed to defined-benefit) pension plans have become increasingly important in many countries as a way to finance retirement. However, in such plans most or all of the financial market risk typically is borne by the individual investors, who are not in a position to be able to manage this risk. The present financial crisis has highlighted once again that this might not be a socially desirable outcome.

On the other hand, the introduction of a guarantee on the return of a pension plan represents a substantial risk for the managing financial institution. The main purpose of the present paper is, firstly, to demonstrate an efficient numerical (semi-analytical) method for valuing equity-linked pension schemes and, secondly, to discuss the qualitative properties of these contracts. We study and compare the pricing (and thus, implicitly, the hedging) of three types of pension schemes with guarantees. These are long term investment plans, in which the investor typically puts in periodic payments of cash over a long period. A proportion of this is invested in an investment fund, while the remainder serves to finance the return guarantee. The resulting equity-linked pension schemes are closely related to equity-linked life insurance contracts, and the results presented here are also applicable to the latter.

In this paper we want to analyze the effect of different guarantee constructions and in each situation to develop possible admissible strategies for the writer of the contract. This is performed by establishing the feasible combinations of a given minimum return guarantee and the fraction of the

\footnotetext{
${ }^{1}$ University of Aarhus, Department of Mathematical Sciences, Ny Munkegade 118, Bldg. 1530, DK-8000 Aarhus C, Denmark, E-mail: atsjan@imf.au.dk

${ }^{2}$ University of Bonn, Department of Finance, Adenauer-Allee 24-42, D-53113 Bonn, Germany, k.sandmann@uni-bonn.de. Part of this work was completed while this author was a Visiting Professor at the University of Technology, Sydney, School of Finance and Economics.

${ }^{3}$ University of Technology, Sydney, School of Finance and Economics, Broadway NSW 2007, Australia, erik.schlogl@uts.edu.au. This author's research is supported under the Australian Research Council's Discovery funding scheme (project number DP0878155).
} 
periodic premium payment set aside to hedge the guarantee.

The related literature focuses primarily on equity-linked life insurance contracts. Ekern and Persson (1996) give an overview of different contract specifications. A first analysis within the context of the arbitrage pricing theory is given by Brennan and Schwartz (1976). They consider the situation of an equity-linked life insurance contract with one up-front premium and use a deterministic model for the term structure of interest rates. Bacinello and Ortu (1994), as well as Nielsen and Sandmann $(1995,1996)$ extend the analysis to include the case of a stochastic interest rate dynamics and periodic premium payments by the insured.

We consider three contract specifications which differ in the type of the guarantee on the outcome of a periodic investment strategy. Our main findings are as follows. For each of these contracts we find a negative (and for two contract specifications also concave) relationship between the participation in the surplus return of the investment strategy and the guarantee level in terms of a minimum rate of return. This property is a consequence of the contract specification, namely the surplus participation and the type of guarantee. It is independent of the financial and non-financial risk involved. As financial risk we consider the price risk of the underlying investment fund and the interest rate risk. As the non-financial risk we explicitly allow for early termination of the contract. We find that the magnitude of the financial risk dominates largely the impact of the non-financial risk. In addition we conclude that the set of feasible contracts changes very smoothly with respect to changes of the non-financial risk. Furthermore, comparing the case allowing for early termination, e.g. due to the death of the pension scheme investor, ${ }^{4}$ with the case involving only a fixed maturity, we find that the two sets of feasible contracts with and without the possibility of early termination are very similar. With respect to the three contract specifications we conclude that in the presence of non-financial risk the "fair" price from the perspective of an underwriter deviates only little from the hedging costs of the financial risks.

\section{Investment Strategy and Guarantees}

The central building block of an equity-linked pension scheme is the specification of an investment strategy. Usually the investment occurs on a periodic basis. Furthermore, the size of the investment amount stays either constant over time or is equal to a constant proportional fraction of the periodic income or earnings by the investor. The periodic investment consists of purchasing shares of a risky fund where the investor can choose among different fund styles. The different fund styles refer to the riskiness of the fund. Purchasing periodically additional shares the investor increases the number of shares and (eventually) builds up a wealth portfolio until the maturity of the investment strategy. Due to the riskiness of the underlying fund, the portfolio value is stochastic. Furthermore, the portfolio value is path-dependent since the number of shares added at each investment date depends on the fund value at this time. This simplified picture at the starting point of our analysis can be formalized as follows: Let $t_{N}=T$ be the maximum maturity of the investment strategy and denote by $\underline{\underline{T}}:=\left\{0=t_{0}<t_{1}<\cdots<t_{N-1}\right\}$, with $t_{N-1}<t_{N}=T$ the set of investment dates. Suppose that at each date $t_{i} \in \underline{\underline{T}}$ the investment amount is given by $k\left(t_{i}\right)$, i.e. the vector $\left(k\left(t_{0}\right), \ldots, k\left(t_{n}\right)\right)$ is equal to the investment sequence until time $t_{n} \in \underline{T}$. To define the portfolio and its value, let $\{S(t)\}_{t \in[0, T]}$ be the stochastic process of the market value of the underlying fund. With this notation the value of the portfolio at time $\left.t \in] t_{0}, t_{N}\right]$ is equal to

$$
\begin{aligned}
P(t, k) & :=\sum_{i=0}^{\min \left\{n^{*}(t), N-1\right\}} k\left(t_{i}\right) \cdot \frac{S(t)}{S\left(t_{i}\right)} \\
\text { with } n^{*}(t) & :=\max \left\{j \in \mathbb{N}_{0} \mid t_{j}<t\right\} .
\end{aligned}
$$

\footnotetext{
${ }^{4}$ Early exercise, i.e. early termination at the discretion of the investor, entails the valuation of American or Bermudan option features. These are the topic of further research and will be considered in a separate, follow-up paper.
} 
The ratio $\frac{k\left(t_{i}\right)}{S\left(t_{i}\right)}$ is equal to the number of additional shares which can be purchased at time $t_{i} \in \underline{\underline{T}}$ by investing the amount $k\left(t_{i}\right)$.

The value of the portfolio is determined by specifying a periodic investment strategy. Assuming no transaction costs or fees, under the absence of arbitrage the present value of the portfolio strategy outcome must equal the present value of the portfolio investment sequence $\left(k\left(t_{0}\right), \ldots, k\left(t_{n}\right)\right)$. An immediate consequence of this simple remark is that any additional guarantee on the outcome of the periodic investment strategy implies additional cost or contributions by the investor. The size of the additional cost obviously depends on the precise form of the guarantee.

In this paper we consider three different but closely related guarantee concepts. To define these different concepts assume for the moment that the terminal date of the contract is ex ante determined as $t_{n} \in \underline{T}$. In other words, we restrict ourselves - at this stage of the paper - to the investment situation and neglect any uncertainty about the terminal date of the contract.

Since any specification of a guarantee implies additional cost, define by $\left(K\left(t_{0}\right), \ldots, K\left(t_{n-1}\right)\right)$ the contribution sequence of the investor. The contribution of the investor consists of the investment (the sequence $k$ ) plus the premium for the guarantee. Our objective is to characterize the relationship between a given pension scheme in terms of its payoff including a guarantee and the corresponding contribution sequence which is sufficient to generate this payoff. In case of a given and certain maturity $t_{n}$ the relationship is based on the following definition

Definition 1 A random payoff $Z\left(t_{n}\right)$ at time $t_{n}$ is called admissible by the contribution sequence $\left(K\left(t_{0}\right), \ldots, K\left(t_{n-1}\right)\right)$ if there exists a self-financing portfolio strategy which generates the payoff $Z\left(t_{n}\right)$ at time $t_{n}$ with initial costs equal to the discounted sum of the contribution, i.e.

$$
\sum_{i=0}^{n-1} K\left(t_{i}\right) \cdot D\left(t_{0}, t_{i}\right),
$$

where $D\left(t_{0}, t_{i}\right)$ denotes the discount factor at time $t_{0}$ with maturity $t_{i}$.

In this sense the payoff $P\left(t_{n}, k\right)$ defined by equation (2.1) is admissible by the contribution sequence $\left(k\left(t_{0}\right), \ldots, k\left(t_{n-1}\right)\right)$. The next step is to discuss different guarantee concepts. Suppose that the contribution of the investor is given by a sequence $\left(K\left(t_{0}\right), \ldots, K\left(t_{n-1}\right)\right)$. Introducing a guarantee implies that the present value of the investment sequence $\left(k\left(t_{0}\right), \ldots, k\left(t_{n-1}\right)\right)$ must be less than the present value of the contribution sequence, with the difference used to finance the guarantee. We assume for simplicity that the connection between the investment amount and the contribution amount at time $t_{i}$ is defined by

$$
k\left(t_{i}\right):=\alpha \cdot K\left(t_{i}\right),
$$

where $\alpha \in[0,1]$ is the investment fraction. In other words, both the investment amount and the additional premium for the guarantee are supplied on a periodic basis. Furthermore, the premium $(1-\alpha) \cdot K\left(t_{i}\right)$ for the guarantee is proportional to the contribution and the proportionality factor is assumed to be time-independent. Under these assumptions, the value of the investment strategy at time $t_{n}$ is equal to

$$
P\left(t_{n}, \alpha \cdot K\right)=\alpha \cdot P\left(t_{n}, K\right)=\alpha \cdot \sum_{i=0}^{n-1} K\left(t_{i}\right) \cdot \frac{S\left(t_{n}\right)}{S\left(t_{i}\right)} .
$$

The different guarantees are now defined with respect to a guaranteed rate of return $g$. In the first case $g$ is guaranteed with respect to the investment amount. In particular, this implies that the minimum return is determined by $\alpha \cdot A\left(t_{n}, g\right)$ with

$$
A\left(t_{n}, g\right):=\sum_{i=0}^{n-1} K\left(t_{i}\right) \cdot \exp \left\{g \cdot\left(t_{n}-t_{i}\right)\right\} .
$$


The payoff at time $t_{n}$ of the investment guarantee scheme ( $I G$-scheme) is determined by

$$
\begin{aligned}
G_{I G}\left(t_{n}, \alpha, g\right) & :=\max \left\{\alpha \cdot P\left(t_{n}, K\right), \alpha \cdot A\left(t_{n}, g\right)\right\} \\
& =\alpha \cdot P\left(t_{n}, K\right)+\alpha \cdot\left[A\left(t_{n}, g\right)-P\left(t_{n}, K\right)\right]^{+} .
\end{aligned}
$$

For any finite guaranteed rate of return $g$ (i.e. any $g>-\infty$ ) the $I G$-scheme is not admissible by the contribution sequence $\left(K\left(t_{0}\right), \ldots, K\left(t_{n-1}\right)\right)$ for $\alpha=1$. For finite $g$ the investment fraction must be less than one.

In the case of the $I G$-scheme, the guarantee refers to the investment amount. The payoff changes if instead the guarantee rate refers to the contribution amount. The minimum guaranteed return is now equal to $A\left(t_{n}, g\right)$ as defined in equation (2.2). The payoff of the contribution guaranteed scheme ( $C G$-scheme) is determined by

$$
\begin{aligned}
G_{C G}\left(t_{n}, \alpha, g\right) & :=\max \left\{\alpha \cdot P\left(t_{n}, K\right), A\left(t_{n}, g\right)\right\} \\
& =\alpha \cdot P\left(t_{n}, K\right)+\left[A\left(t_{n}, g\right)-\alpha \cdot P\left(t_{n}, K\right)\right]^{+} .
\end{aligned}
$$

The payoff of both schemes coincide in case of an investment fraction $\alpha$ equal to one, but are not admissible in this case by the contribution sequence $\left(K\left(t_{0}\right), \ldots, K\left(t_{n-1}\right)\right)$. Furthermore both schemes are defined in a natural way as floors for the underlying investment payoff. Basically, the payoff is characterized by a long position in the investment strategy plus an additional put option. As a consequence of the periodic investment, the corresponding put options are closely related to an Asian-type put option.

The definition of the third scheme looks different at first glance. In this case the construction of the payoff is equal to a fixed guaranteed amount plus a participation in the surplus of the investment payoff over the guaranteed amount. The guaranteed amount is again given by the sum of the contributions compounded with a rate of return $g$, i.e. by the amount $A\left(t_{n}, g\right)$. Since the investor additionally receives a surplus proportional to the positive difference between the return of the periodic investment strategy and the guaranteed amount, this case is called the surplus participation scheme, $S P$-scheme. The payoff of the $S P$-scheme is defined by:

$$
\begin{aligned}
G_{S P}\left(t_{n}, \alpha, g\right) & :=A\left(t_{n}, g\right)+\alpha \cdot\left[P\left(t_{n}, K\right)-A\left(t_{n}, g\right)\right]^{+} \\
& =\alpha \cdot P\left(t_{n}, K\right)+(1-\alpha) \cdot A\left(t_{n}, g\right)+\alpha \cdot\left[A\left(t_{n}, g\right)-P\left(t_{n}, K\right)\right]^{+} .
\end{aligned}
$$

Since the payoff of all three different schemes is a monotonic function of the corresponding investment share and the interest rate guarantee, a first implication with respect to the set of admissible payoffs is given by the following Proposition.

Proposition 2.1 Suppose that the payoffs of the $I G$-scheme with parameters $\left(\alpha_{I G}, g_{I G}\right)$, the $C G$ scheme with parameters $\left(\alpha_{C G}, g_{C G}\right)$ and the $S P$-scheme with parameters $\left(\alpha_{S P}, g_{S P}\right)$ are admissible by the same contribution sequence.

(i) In the case of identical interest rate guarantees, i.e.

$$
g_{I G}=g_{C G}=g_{S P}
$$

the investment fraction of the $I G$-scheme is bounded from below by the investment fraction of the CG-scheme, which is bounded from below by the one of the SP-scheme, i.e.

$$
\alpha_{I G} \geq \alpha_{C G} \geq \alpha_{S P}
$$

(ii) In the case of identical investment fractions, i.e.

$$
\alpha_{I G}=\alpha_{C G}=\alpha_{S P}
$$

the interest rate guarantee of the IG-scheme is bounded from below by the interest rate guarantee of the CG-scheme, which is bounded from below by the one of the SP-scheme, i.e.

$$
g_{I G} \geq g_{C G} \geq g_{S P}
$$


Proof: Consider first the payoff of the $I G$ - and $C G$-scheme. In the case of an identical investment fraction $\alpha \in[0,1]$ and an identical guaranteed rate of return $g$, the payoff of the $I G$-scheme is dominated by the one of the $C G$-scheme since

$$
\alpha \cdot P\left(t_{n}, K\right)+\alpha \cdot\left[A\left(t_{n}, g\right)-P\left(t_{n}, K\right)\right]^{+} \leq \alpha \cdot P\left(t_{n}, K\right)+\left[A\left(t_{n}, g\right)-\alpha \cdot P\left(t_{n}, K\right)\right]^{+} .
$$

Given identical parameters, we therefore can conclude that under no-arbitrage the present value of the generating self-financing strategy of the the $C G$-scheme is weakly bounded from below by the present value of the self-financing strategy generating the payoff of the $I G$-scheme. Furthermore the payoff of the $C G$-scheme is increasing in $\alpha$ and $g$. By assumption both payoffs must be admissible by the same contribution sequence. As a consequence the guaranteed rate of return $g_{C G}$ of the $C G$-scheme must be less than or equal to the guaranteed rate of return $g_{I G}$ of the $I G$-scheme if the investment fractions are identical. Conversely, the investment fraction $\alpha_{C G}$ of the $C G$-scheme must less than or equal to the investment fraction $\alpha_{I G}$ of the $I G$-scheme if the guaranteed rates of return are identical.

To relate the $C G$ - and the $S P$-scheme, as before the argument is based a pathwise inequality of the payoffs. More precisely, for identical parameters the following inequality holds

$$
\begin{aligned}
& \alpha \cdot P\left(t_{n}, K\right)+\left[A\left(t_{n}, g\right)-\alpha \cdot P\left(t_{n}, K\right)\right]^{+} \\
= & \alpha \cdot P\left(t_{n}, K\right)+\left[(1-\alpha) \cdot A\left(t_{n}, g\right)+\alpha \cdot A\left(t_{n}, g\right)-\alpha \cdot P\left(t_{n}, K\right)\right]^{+} \\
\leq & \alpha \cdot P\left(t_{n}, K\right)+(1-\alpha) \cdot A\left(t_{n}, g\right)+\alpha \cdot\left[A\left(t_{n}, g\right)-P\left(t_{n}, K\right)\right]^{+} .
\end{aligned}
$$

In case of identical parameters the payoff of the $C G$-scheme is weakly dominated by the payoff of the $S P$-scheme, thus the inequalities in the Proposition follow.

The inequalities in Proposition 2.1 are strict if in addition the support of the distribution of the market value of the underlying fund is equal to $\mathbb{R}_{+}$. Furthermore, the following result with respect to the investment fraction is again a direct consequence of the monotonicity in the payoff structure for all schemes:

Proposition 2.2 For a fixed contribution sequence consider the set of parameters $(\alpha, g)$ such that the corresponding IG-scheme (CG-scheme, SP-scheme) is admissible. As a function of the guaranteed rate of return, the investment fraction $\alpha(g)$ is decreasing.

The guaranteed payment of all three schemes is determined by $A\left(t_{n}, g\right)$ as defined by equation (2.2). As a function of the guaranteed rate of return $g$, the guaranteed payment converges to zero if the rate approaches minus infinity. This limiting case reflects the situation without a guarantee. In this sense all three schemes are generalizations of the pure investment situation. The investment share of an admissible payoff in this case is equal to one. On the other hand, consider the case of an investment share equal to zero. The situation for the $I G$-scheme is quite trivial since for $\alpha=0$ the payoff is equal to zero (degenerate case). Thus the payoff is admissible by setting the contribution sequence equal to zero. Obviously this is not the only solution satisfying Definition 1 . Setting the investment share $\alpha$ equal to zero implies that the payoff of the $C G$-Scheme and the $S P$-scheme are both identical and equal to

$$
A\left(t_{n}, g\right):=\sum_{i=0}^{n-1} K\left(t_{i}\right) \cdot \exp \left\{g \cdot\left(t_{n}-t_{i}\right)\right\} .
$$

Furthermore the payoff of both schemes is deterministic. For $\alpha=0$ both schemes are identical to a fixed interest rate contract with periodic investments. The payoff is admissible by the contribution sequence $\left(K\left(t_{0}\right), \ldots, K\left(t_{n-1}\right)\right)$ iff

$$
D\left(t_{0}, t_{n}\right) \cdot\left(\sum_{i=0}^{n-1} K\left(t_{i}\right) \cdot \exp \left\{g \cdot\left(t_{n}-t_{i}\right)\right\}\right)=\sum_{i=0}^{n-1} K\left(t_{i}\right) \cdot D\left(t_{0}, t_{i}\right)
$$


For a given contribution sequence $\left(K\left(t_{0}\right), \ldots, K\left(t_{n-1}\right)\right)$ equation (2.6) defines the implied guaranteed rate of return such that the payoff is admissible. As a simple case consider identical contributions over time, i.e. $K\left(t_{i}\right)=K, \forall i=0, \ldots, n-1$. The condition on the guaranteed rate of return can be simplified to

$$
\left(\sum_{i=0}^{n-1} \exp \left\{g \cdot\left(t_{n}-t_{i}\right)\right\}\right)=D\left(t_{0}, t_{n}\right)^{-1} \cdot\left(\sum_{i=0}^{n-1} D\left(t_{0}, t_{i}\right)\right) .
$$

Since the sum of all zero coupon bonds is known as the annuity factor and by dividing by the long term bond we get the forward value of the annuity factor, the solution $g^{*}$ of equation (2.7) can be interpreted as the forward annuity yield. In the case of a flat initial term structure with time independent yield $y$, i.e. $D\left(t_{0}, t_{i}\right)=\exp \left(-y \cdot\left(t_{i}-t_{0}\right)\right\}$ the forward annuity yield $g^{*}$ is equal to $y$. As a conclusion we have the following result for the $C G$ - and the $S P$-scheme:

Proposition 2.3 Consider a contribution sequence until time $t_{n}$ with constant contributions, i.e. $K\left(t_{i}\right)=K, \forall i=0, \ldots, n-1$. The investment fraction of an admissible payoff for the $C G$-scheme (SP-scheme) is monotonically decreasing in the guaranteed rate of return $g$ with

$$
\alpha(g)= \begin{cases}>0 & \text { if } g<g^{*} \\ =0 & \text { if } g=g^{*} \\ <0 & \text { if } g>g^{*}\end{cases}
$$

where $g^{*}$ is equal to the forward annuity yield, i.e. a solution to

$$
\left(\sum_{i=0}^{n-1} \exp \left\{g \cdot\left(t_{n}-t_{i}\right)\right\}\right)=D\left(t_{0}, t_{n}\right)^{-1} \cdot\left(\sum_{i=0}^{n-1} D\left(t_{0}, t_{i}\right)\right) .
$$

\section{Insurance and Financial Risk}

So far the contract specification is not yet complete. Unlike a pure financial contract, the termination date of the pension contract is potentially not equal to the maturity $t_{N}=T$. Instead the contract may terminate at any time $t_{i} \in \underline{\underline{T}}$ prior to $t_{N}$, e.g. due to the death of the investor. The final date $t_{N}=T$ is only the last possible date the contract matures. Further rules on the possibility of early termination may be related to different national legislation and each of them will have an impact on the analysis of the contract situation. In our present context the termination date of the contract is a random variable $\tau$. The distribution of $\tau$ depends on the death- and survival probability of the investor and in the simplest case is determined by this probability (but in general may depend on other factors). In notation of the mortality distribution let us denote by $\pi_{x}(t)$ the ex-ante density function of the early termination for an investor aged $x$ at time $t_{0}$. The ex-ante early termination distribution is defined by:

$$
\begin{aligned}
& \int_{t_{0}}^{t} \pi_{x}(u) d u=: \quad \operatorname{prob}\left[\text { an investor aged } x\left(\text { at time } t_{0}\right)\right. \text { terminates the pension } \\
& \text { contract within the period } \left.\left.] t_{0}, t\right]\right] \\
& 1-\int_{t_{0}}^{t} \pi_{x}(u) d u=: \quad \operatorname{prob}\left[\text { an investor aged } x \text { (at time } t_{0}\right) \text { does not terminate } \\
& \text { the pension contract prior } t] \text {. }
\end{aligned}
$$

As in the case of the mortality distribution, we assume that the data available to the policy underwriter allows him to estimate these densities. More precisely, we assume that the policy issuer, at time $t_{0}$, knows the ex-ante probability density functions of early contract termination for an investor aged $\mathrm{x} \forall x$. Within the numerical analysis in Section 6 we will continue to specify the early exercise 
distribution by applying a Wang-Transformation to a Makeham distribution. For the moment, it is sufficient to assume that the ex ante density is given.

The benefit side of the equity-linked pension contract is now determined by the termination time $\tau$, which is determined by the termination distribution and the pension scheme chosen. More precisely we define the an equity-linked pension contract as follows

Definition 2 Consider a contribution sequence $\left(K\left(t_{0}\right), \ldots, K\left(t_{N-1}\right)\right)$ and denote by the index $c \in$ $\{I G, C G, S P\}$ the corresponding payoff-scheme as defined in Section 2. A contract with the following payoffs is called an type $c$ equity linked pension scheme with investment share $\alpha$, guaranteed rate of return $g$ and ex-ante contribution sequence $\left(K\left(t_{0}\right), \ldots, K\left(t_{N-1}\right)\right)$ if the following holds:

- If termination occurs prior to the maturity $T$ at time $\tau \in] 0, T[$, the investor receives at time $\tau$ the payoff

$$
G_{c}(\tau, \alpha, g) \text { for } c \in\{I G, C G, S P\}
$$

and in this case the contribution sequence is equal to $\left(K\left(t_{0}\right), \ldots, K\left(t_{M}\right)\right)$, where $t_{M}$ is the last investment date before time $\tau$, i.e

$$
\left.M:=\min \left\{n^{*}(\tau), N-1\right\}\right\} .
$$

- If the contract has not been terminated prior to $T$, the contract matures at time $T$ with a payoff defined by

$$
G_{c}(T, \alpha, g) \text { for } c \in\{I G, C G, S P\} .
$$

and in this case the contribution sequence is $\left(K\left(t_{0}\right), \ldots, K\left(t_{N-1}\right)\right)$.

In addition to the termination distribution, the equity-linked pension contract is influenced by two types of financial risks. Since the benefit to the investor is a function of the value of the investment portfolio, the dynamics of the underlying mutual fund are involved. In addition, the contract is of long term and therefore the interest rate risk must be considered. These financial aspects are included in the analysis by a complete and arbitrage-free model of the financial market. Let $Q^{*}$ be the unique equivalent martingale measure, such that, with respect to a filtered probability space $\left(\Omega, \mathbb{F}, Q^{*},\left\{\mathbb{F}_{t}\right\}\right)$, the discounted price processes of the mutual fund $\{S(t)\}_{t}$ and the discounted price processes of all the zero coupon bonds $\{D(t, \tau)\}_{t \in\left[t_{0}, \tau\right]}$ with maturity $\tau \in \mathbb{R}_{\geq 0}$ are martingales:

$$
\begin{array}{rlrl}
S(t) & =E_{Q^{*}}\left[\beta_{t, \bar{t}}^{-1} \cdot S(\bar{t}) \mid \mathbb{F}_{t}\right] \quad \forall \bar{t} \geq t, \\
D(t, \tau) & =E_{Q^{*}}\left[\beta_{t, \bar{t}}^{-1} \cdot D(\bar{t}, \tau) \mid \mathbb{F}_{t}\right] \quad \forall \bar{t} \in[t, \tau], \forall \tau \geq \bar{t}, \\
D(\tau, \tau) & =1 \quad Q^{*} \text { a.s. } &
\end{array}
$$

In addition to the martingale property of the discounted price processes we assume enough regularity, so that for each fixed $\tau \in \mathbb{R}_{>0}$ there exists a unique $\tau$-forward risk adjusted measure $Q^{\tau}$ defined by

$$
\left.\frac{d Q^{\tau}}{d Q^{*}}\right|_{t}=\frac{\beta_{t_{0}, t}^{-1} \cdot D(t, \tau)}{E_{Q^{*}}\left[\beta_{t_{0}, t}^{-1} \cdot D(t, \tau) \mid \mathbb{F}_{t_{0}}\right]} .
$$

The change of measure technique implies that the forward prices of the financial assets are martingales under the appropriate forward risk adjusted measure, i.e.

$$
\begin{aligned}
S(t) & =D(t, \tau) \cdot E_{Q^{\tau}}\left[\frac{S(\tau)}{D(\tau . \tau)} \mid \mathbb{F}_{t}\right] & \forall \tau \geq t \geq t_{0}, \\
D(t, T) & =D(t, \tau) \cdot E_{Q^{\tau}}\left[\frac{D(\tau, T)}{D(\tau, \tau)} \mid \mathbb{F}_{t}\right] & \forall T \geq \tau \geq t \geq t_{0} .
\end{aligned}
$$

This is the usual and standard setup of a complete and arbitrage-free financial market model including interest rate risk. At this point in the discussion we do not need to specify the volatility 
structure. The general results, with respect to the relationship between the investment share and the rate of return guarantee do not depend on any more specific assumptions about the volatility structure. Nevertheless, for the numerical analysis to be performed, we will assume a special framework with deterministic volatilities. For further details concerning the financial market model we refer to Geman, El Karoui and Rochet (1995). The following Proposition summarizes some useful results with respect to the expected value of the underlying fund and the termination distribution.

Proposition 3.1 Consider a financial market model satisfying the relationships (3.1) to (3.3) and a deterministic contribution sequence $\left(K\left(t_{0}\right), \ldots, K\left(t_{N-1}\right)\right)$. For $t_{N}=T \geq t>t_{0}$

$$
\begin{aligned}
E_{Q^{t}}\left[\sum_{i=0}^{n^{*}(t)} \frac{S(t)}{S\left(t_{i}\right)} \mid \mathbb{F}_{t_{0}}\right] & =\sum_{i=0}^{n^{*}(t)} \frac{D\left(t_{0}, t_{i}\right)}{D\left(t_{0}, t\right)} \\
\int_{t_{0}}^{T}\left(\sum_{i=0}^{n^{*}(u)} K\left(t_{i}\right) \cdot D\left(t_{0}, t_{i}\right)\right) \cdot \pi_{x}(u) d u & +\left(\sum_{i=0}^{N-1} K\left(t_{i}\right) \cdot D\left(t_{0}, t_{i}\right)\right) \cdot\left(1-\int_{t_{0}}^{T} \pi_{x}(u) d u\right) \\
& =\sum_{i=0}^{N-1}\left(K\left(t_{i}\right) \cdot D\left(t_{0}, t_{i}\right) \cdot\left(1-\int_{t_{0}}^{t_{i}} \pi_{x}(u) d u\right)\right) .
\end{aligned}
$$

Proof: Denote by $Q^{*}$ the spot risk neutral measure associated with taking the continuously compounded savings account,

$$
\beta_{t, T}=\exp \left\{\int_{t}^{T} r(s) d s\right\}
$$

as the numeraire (where $r(s)$ denotes the continuously compounded short rate of interest at time $s)$. The first expectation under the forward measure $Q^{t}$ is $\forall i$ with $t_{i} \leq t$ given by:

$$
\begin{aligned}
E_{Q^{t}}\left[\frac{S(t)}{S\left(t_{i}\right)} \mid \mathbb{F}_{t_{0}}\right] & =\frac{1}{D\left(t_{0}, t\right)} \cdot E_{Q^{*}}\left[\beta_{t_{0}, t}^{-1} \cdot \frac{S(t)}{S\left(t_{i}\right)} \mid \mathbb{F}_{t_{0}}\right] \\
& =\frac{1}{D\left(t_{0}, t\right)} \cdot E_{Q^{*}}\left[\beta_{t_{0}, t_{i}}^{-1} \cdot \frac{1}{S\left(t_{i}\right)} E_{Q^{*}}\left[\beta_{t_{i}, t}^{-1} \cdot S(t) \mid \mathbb{F}_{t_{i}}\right] \mid \mathbb{F}_{t_{0}}\right] \\
& =\frac{1}{D\left(t_{0}, t\right)} \cdot E_{Q^{*}}\left[\beta_{t_{0}, t_{i}}^{-1} \cdot \frac{S\left(t_{i}\right)}{S\left(t_{i}\right)} \mid \mathbb{F}_{t_{0}}\right]=\frac{D\left(t_{0}, t_{i}\right)}{D\left(t_{0}, t\right)} \quad \forall t \geq t_{i} \geq t_{0}
\end{aligned}
$$

The second equality is justified by the following calculation:

$$
\begin{aligned}
& \int_{t_{0}}^{T}\left(\sum_{i=0}^{n^{*}(u)} K\left(t_{i}\right) \cdot D\left(t_{0}, t_{i}\right)\right) \cdot \pi_{x}(u) d u \\
= & \sum_{j=0}^{N-1} \int_{t_{j}}^{t_{j+1}}\left(\sum_{i=0}^{j} K\left(t_{i}\right) \cdot D\left(t_{0}, t_{i}\right)\right) \cdot \pi_{x}(u) d u \\
= & \sum_{j=0}^{N-1}\left(\left(\sum_{i=0}^{j} K\left(t_{i}\right) \cdot D\left(t_{0}, t_{i}\right)\right) \cdot \int_{t_{j}}^{t_{j+1}} \pi_{x}(u) d u\right) \\
= & \sum_{j=0}^{N-1}\left(K\left(t_{j}\right) \cdot D\left(t_{0}, t_{j}\right) \cdot \int_{t_{j}}^{T} \pi_{x}(u) d u\right) \\
= & \sum_{j=0}^{N-1}\left(K\left(t_{j}\right) \cdot D\left(t_{0}, t_{j}\right) \cdot\left(1-\int_{t_{0}}^{t_{j}} \pi_{x}(u) d u-1+\int_{t_{0}}^{T} \pi_{x}(u) d u\right)\right)
\end{aligned}
$$




\section{The Set of on Average Admissible Pension Schemes}

In Section 2 we introduced the notion of an admissible investment contract. This notion deviates only in a minor way from the usual no-arbitrage pricing principle. Instead of the initial starting costs we concentrate on periodic contributions. Even if the no-arbitrage price of a given random payoff is unique, the set of admissible contribution sequences is not singleton. For option pricing problems under the completeness assumption this is not the natural approach. Nevertheless the setup in case of an equity-linked pension contract is different. First, the contributions by the investor occur in most applications periodically. Second, even under assumption of a complete financial market, we have to consider a potentially incomplete market situation due to the possibility of early termination of the contract. In general there exists no self-financing portfolio strategy which replicates the payoff of the pension contract on a per-contract basis when one allows for early termination due to an external source of risk.

As early termination is closely related to the life insurance risk we assume that the equivalence principle can be applied with respect to this risk. That is, we assume that the risk related to early termination can be diversified by the policy holder within the population of the investors.

Obviously, the financial risk cannot be diversified by this risk management technique. The assumption of a complete market implies that any payoff at a given time $t$ can be perfectly hedged by a self-financing portfolio strategy on the financial market. Furthermore, the initial value of this portfolio strategy is equal to the expected discounted payoff under the unique martingale measure. Since the equity-linked pension scheme does not allow the separation of the payoff into a pure financial and a pure insurance contract, we cannot apply these two principles separately. Therefore the solution concept has to combine both risk management strategies. As Brennan and Schwartz (1976), Bacinello and Ortu (1994) as well as Nielsen and Sandmann (1995, 1996), we assume that the insurance risk and the financial risks are independent. With these remarks we are now ready to extend the definition of an admissible payoff to the situation of a pension scheme.

Definition 3 Consider an equity-linked pension scheme of type $c \in\{I G, C G, S P\}$ as defined by Definition 2 and an investor at age $x>0$.

- The type c-pension scheme with investment share $\alpha$ and guaranteed rate of return $g$ is called admissible on average for an investor aged $x$ by a non-negative periodic contribution sequence $\left(K^{*}\left(t_{0}\right), \ldots, K^{*}\left(t_{N}\right)\right)$, if the contribution sequence is a solution to

$$
\begin{aligned}
& \sum_{i=0}^{N-1} K^{*}\left(t_{i}\right) \cdot D\left(t_{0}, t_{i}\right) \cdot\left(1-\int_{t_{0}}^{t_{i}} \pi_{x}(u) d u\right) \\
= & \int_{t_{0}}^{t_{N}} D\left(t_{0}, u\right) \cdot E_{Q^{u}}\left[G_{c}(u, \alpha, g) \mid \mathbb{F}_{0}\right] \cdot \pi_{x}(u) d u \\
& +D\left(t_{0}, t_{N}\right) \cdot E_{Q^{t_{N}}}\left[G_{c}\left(t_{N}, \alpha, g\right) \mid \mathbb{F}_{0}\right] \cdot\left(1-\int_{t_{0}}^{t_{N}} \pi_{x}(u) d u\right) .
\end{aligned}
$$

The contribution sequence $\left(K^{*}\left(t_{0}\right), \ldots, K^{*}\left(t_{N-1}\right)\right)$ is called a fair premium of the type cpension scheme for the investor at age $x$.

- For a given contribution sequence $\left(K\left(t_{0}\right), \ldots, K\left(t_{N-1}\right)\right)$ define

$$
I_{x, c}\left(K\left(t_{0}\right), \ldots, K\left(t_{N-1}\right)\right) \subset \mathbb{R}^{2}
$$

as the set of tuples $(\alpha, g)$ such that the type c-pension scheme with investment share $\alpha$ and guaranteed rate of return $g$ is admissible on average for an investor aged $x$ by the periodic contribution sequence $\left(K\left(t_{0}\right), \ldots, K\left(t_{N-1}\right)\right)$.

To derive the existence of a fair premium and furthermore to characterize the set

$$
I_{x, c}\left(K\left(t_{0}\right), \ldots, K\left(t_{N-1}\right)\right)
$$


for a given contribution, two extreme cases have to be considered. The first case is given for the choice $\alpha=0$. This corresponds to a pension scheme with a deterministic payoff.

In case of an type $I G$-pension scheme with investment share equal to zero the situation is quite easy, since the payoff is always equal to zero. Therefore the expected discounted benefit to the investor, given by the right hand side of equation (4.1), is equal to zero. The condition on the fair premium implies that the left hand side of equation (4.1) must be zero as well. If we restrict ourself to non-negative contribution sequences the unique solution is given by the zero contribution sequence. If instead negative contributions are permitted the set of solutions is no longer a singleton. The left hand side of equation (4.1) can be interpreted as the termination adjusted weighted sum of the contributions with weights determined by the discounted non-termination probability.

Proposition 4.1 The $C G$ - or $S P$-pension scheme with investment share $\alpha=0$ and guaranteed rate of return $g^{*}$ is on average admissible by the contribution sequence $K\left(t_{0}\right), \ldots, K\left(t_{N-1}\right)$, i.e.

$$
(0, g *) \in I_{x, c}\left(K\left(t_{0}\right), \ldots, K\left(t_{N-1}\right)\right) \text { for } c \in\{C G, S P\}
$$

if the guaranteed rate of return $g^{*}$ is the solution of

$$
\begin{aligned}
& \sum_{i=0}^{N-1}\left(K\left(t_{i}\right) \cdot D\left(t_{0}, t_{i}\right) \cdot\left(1-\int_{t_{0}}^{t_{i}} \pi_{x}(u) d u\right)\right) \\
= & \int_{t_{0}}^{T}\left(\sum_{i=0}^{n^{*}(u)} K\left(t_{i}\right) \cdot D\left(t_{0}, u\right) \cdot \exp \left\{g \cdot\left(u-t_{i}\right)\right\}\right) \cdot \pi_{x}(u) d u \\
& +\left(\sum_{i=0}^{N-1} K\left(t_{i}\right) \cdot D\left(t_{0}, t_{N}\right) \cdot \exp \left\{g \cdot\left(t_{N}-t_{i}\right)\right\}\right) \cdot\left(1-\int_{t_{0}}^{t_{N}} \pi_{x}(u) d u\right) .
\end{aligned}
$$

If in particular the initial term structure is flat then the unique solution is independent of the contribution sequence given by the yield $y$, i.e.

$$
g^{*}=y \text { with } D\left(t_{0}, t_{i}\right)=\exp \left\{-y \cdot\left(t_{i}-t_{0}\right)\right\} \forall t_{i} \in \underline{\underline{T}} .
$$

If the contribution is constant over time the guaranteed rate of return $g^{*}$ satisfies

$$
g^{*} \in\left[f_{\min }\left(t_{0}\right), f_{\max }\left(t_{0}\right)\right],
$$

where the minimum and maximum initial forward yield are defined by

$$
\begin{gathered}
\exp \left\{-f_{\text {min }}\left(t_{0}\right) \cdot\left(u-t_{i}\right)\right\} \geq \frac{D\left(t_{0}, u\right)}{D\left(t_{0}, t_{i}\right)} \quad \forall T \geq u>t_{i} \text { and } \forall t_{i} \in \underline{\underline{T}} \\
\exp \left\{-f_{\text {max }}\left(t_{0}\right) \cdot\left(u-t_{i}\right)\right\} \leq \frac{D\left(t_{0}, u\right)}{D\left(t_{0}, t_{i}\right)} \quad \forall T \geq u>t_{i} \text { and } \forall t_{i} \in \underline{\underline{T}} .
\end{gathered}
$$

Proof: If the investment share is equal to zero the payoff of the $C G$ - and the $S P$-scheme is deterministic and in both cases equal to

$$
G_{C G}(u, 0, g)=G_{S P}(u, 0, g)=A(u, g):=\sum_{i=0}^{n^{*}(u)} K\left(t_{i}\right) \cdot \exp \left\{g \cdot\left(u-t_{i}\right)\right\},
$$

with $n^{*}(u)=\max \left\{j \in \mathbb{N}_{0} \mid t_{j}<u\right\}$. The expected discounted benefit for the investor is thus equal 
to

$$
\begin{aligned}
& \int_{t_{0}}^{T} D\left(t_{0}, u\right) \cdot A(u, g) \cdot \pi_{x}(u) d u+D\left(t_{0}, t_{N}\right) \cdot A\left(t_{N}, g\right) \cdot\left(1-\int_{t_{0}}^{t_{N}} \pi_{x}(u) d u\right) \\
= & \int_{t_{0}}^{T} D\left(t_{0}, u\right) \cdot\left(\sum_{i=0}^{n^{*}(u)} K\left(t_{i}\right) \cdot \exp \left\{g \cdot\left(u-t_{i}\right)\right\}\right) \cdot \pi_{x}(u) d u \\
& +D\left(t_{0}, t_{N}\right) \cdot\left(\sum_{i=0}^{N-1} K\left(t_{i}\right) \cdot \exp \left\{g \cdot\left(t_{N}-t_{i}\right)\right\}\right) \cdot\left(1-\int_{t_{0}}^{t_{N}} \pi_{x}(u) d u\right) \\
= & \int_{t_{0}}^{T}\left(\sum_{i=0}^{n^{*}(u)} K\left(t_{i}\right) \cdot D\left(t_{0}, u\right) \cdot \exp \left\{g \cdot\left(u-t_{i}\right)\right\}\right) \cdot \pi_{x}(u) d u \\
& +\left(\sum_{i=0}^{N-1} K\left(t_{i}\right) \cdot D\left(t_{0}, t_{N}\right) \cdot \exp \left\{g \cdot\left(t_{N}-t_{i}\right)\right\}\right) \cdot\left(1-\int_{t_{0}}^{t_{N}} \pi_{x}(u) d u\right),
\end{aligned}
$$

which is equal to the expected discounted contribution if the guaranteed rate of return $g$ satisfies the above condition. In the case of a flat term structure with yield $y$ and by setting the guaranteed rate of return $g$ equal to the yield we have

$$
D\left(t_{0}, u\right) \cdot \exp \left\{g \cdot\left(u-t_{i}\right)\right\}=D\left(t_{0}, t_{i}\right) \cdot \exp \left\{(g-y) \cdot\left(u-t_{i}\right)\right\}=D\left(t_{0}, t_{i}\right) .
$$

In this particular case the expected discounted benefit can be simplified to

$$
\begin{aligned}
& \int_{t_{0}}^{T}\left(\sum_{i=0}^{n^{*}(u)} K\left(t_{i}\right) \cdot D\left(t_{0}, t_{i}\right)\right) \cdot \pi_{x}(u) d u+\left(\sum_{i=0}^{N-1} K\left(t_{i}\right) \cdot D\left(t_{0}, t_{i}\right)\right) \cdot\left(1-\int_{t_{0}}^{t_{N}} \pi_{x}(u) d u\right) \\
= & \sum_{i=0}^{N-1}\left(K\left(t_{i}\right) \cdot D\left(t_{0}, t_{i}\right) \cdot\left(1-\int_{t_{0}}^{t_{i}} \pi_{x}(u) d u\right)\right),
\end{aligned}
$$

In the case of a constant contribution sequence but not necessarily flat term structure the following inequalities are satisfied

$$
\begin{aligned}
& D\left(t_{0}, u\right) \cdot \exp \left\{g \cdot\left(u-t_{i}\right)\right\}=D\left(t_{0}, t_{i}\right) \cdot \frac{D\left(t_{0}, u\right)}{D\left(t_{0}, t_{i}\right)} \cdot \exp \left\{g \cdot\left(u-t_{i}\right)\right\} \\
&\left\{\begin{array}{ll}
<D\left(t_{0}, t_{i}\right) \forall u & \text { if } g<f_{\text {min }} \\
>D\left(t_{0}, t_{i}\right) \forall u & \text { if } g>f_{\text {max }}
\end{array} .\right.
\end{aligned}
$$

For $g<f_{\min }$ the expected discounted benefit is less than the expected discounted contribution. On the other hand $g>f_{\max }$ implies that the expected discounted benefit is larger than the expected discounted contribution. Since the expected discounted benefit is a continuous function of the guaranteed rate of return there exists a unique rate $g \in\left[f_{\min }, f_{\max }\right]$ which implies equality.

Proposition 4.2 covers the situation without any guaranteed payments by the insurer.

Proposition 4.2 For $c \in\{I G, C G, S P\}$ the c-pension scheme with investment share $\alpha=1$ and contribution sequence $K\left(t_{0}\right), \ldots, K\left(t_{N-1}\right)$ is admissible if and only if there exists no guarantee, i.e guaranteed rate of return is equal to $-\infty$

$$
(1,-\infty) \in I_{x, c}\left(K\left(t_{0}\right), \ldots, K\left(t_{N-1}\right)\right)
$$

Proof: For all three pension schemes the payoff in case of an investment share equal to one is bounded from below by the portfolio value, i.e.

$$
G_{c}(u, 1, g) \geq P(u, K)=\sum_{i=0}^{\min \left\{n^{*}(u), N-1\right\}} K\left(t_{i}\right) \cdot \frac{S(u)}{S\left(t_{i}\right)} \quad \forall g \in \mathbb{R} .
$$


Therefore the expected discounted benefit is bounded from below by

$$
\begin{gathered}
\int_{t_{0}}^{t_{N}} D\left(t_{0}, u\right) \cdot E_{Q^{t_{u}}}\left[G_{c}(u, 1, g) \mid \mathbb{F}_{0}\right] \cdot \pi_{x}(u) d u \\
+D\left(t_{0}, t_{N}\right) \cdot E_{Q^{t_{N}}}\left[G_{c}\left(t_{N}, 1, g\right) \mid \mathbb{F}_{0}\right] \cdot\left(1-\int_{t_{0}}^{t_{N}} \pi_{x}(u) d u\right) \\
\geq \quad \int_{t_{0}}^{t_{N}} D\left(t_{0}, u\right) \cdot E_{Q^{t_{u}}}\left[\sum_{i=0}^{n^{*}(u)} K\left(t_{i}\right) \cdot \frac{S(u)}{S\left(t_{i}\right)} \mid \mathbb{F}_{0}\right] \cdot \pi_{x}(u) d u \\
\quad+D\left(t_{0}, t_{N}\right) \cdot E_{Q^{t_{N}}}\left[\sum_{i=0}^{N-1} K\left(t_{i}\right) \cdot \frac{S\left(t_{N}\right)}{S\left(t_{i}\right)} \mid \mathbb{F}_{0}\right] \cdot\left(1-\int_{t_{0}}^{t_{N}} \pi_{x}(u) d u\right) \\
=\int_{t_{0}}^{t_{N}} D\left(t_{0}, u\right) \cdot \sum_{i=0}^{n^{*}(u)} K\left(t_{i}\right) \cdot \frac{D\left(t_{0}, t_{i}\right)}{D\left(t_{0}, u\right)} \cdot \pi_{x}(u) d u \\
+\int_{t_{0}}^{N-1} \sum_{i=0}^{N-1} K\left(t_{0}, t_{N}\right) \cdot \sum_{i=0}^{t_{N}} K\left(t_{i}\right) \cdot \frac{D\left(t_{0}, t_{i}\right)}{D\left(t_{0}, t_{N}\right)} \cdot\left(1-\int_{t_{0}}^{t_{N}} \pi_{x}(u) d u\right) \\
\sum_{i=0}^{N-1}\left(K\left(t_{i}\right) \cdot D\left(t_{0}, t_{i}\right) \cdot\left(1-\pi_{x}(u) d u+\sum_{t_{0}}^{n^{*}(u)} \pi_{x}(u) d u\right)\right) \cdot
\end{gathered}
$$

where the equalities are consequences of Proposition 3.1. Since the payoff is an increasing function of the guaranteed rate of return $g$ the pension scheme is admissible if and only if no guaranteed payment is contracted.

So far our discussion of the limiting cases implies that for a given contribution sequence $K\left(t_{0}\right), \ldots$, $K\left(t_{N-1}\right)$ the set of admissible contacts $I_{x, c}\left(K\left(t_{0}\right), \ldots, K\left(t_{N-1}\right)\right)$ is not empty. For the remaining part of the discussion consider a fixed contribution sequence. More precisely, we study the investment fraction $\alpha$ as a function of the guaranteed rate of return $g$ such that $(\alpha(g), g) \in I_{x, c}(K)$. To derive the functional relationship between these two contract parameters we have to reconsider the definition of a fair premium. The fair premium concept requires equality between the expected discounted contribution and the expected discounted benefit. The expected discounted contribution is a function of the contribution sequence, the discounting factors and the distribution of the termination date. This part of the solution equation can be summarized by a term $B_{1}$, which is independent of the investment fraction and the guaranteed rate of return, i.e.

$$
B_{1}:=\sum_{i=0}^{N-1} K\left(t_{i}\right) \cdot D\left(t_{0}, t_{i}\right) \cdot\left(1-\int_{t_{0}}^{t_{i}} \pi_{x}(u) d u\right) .
$$

The expected discounted benefit of each pension scheme can be decomposed into three different parts. The first part is equal to the expected discounted portfolio value. As already used earlier, Proposition 3.1 implies that this part is equal to $B_{1}$ as well, i.e.

$$
\begin{aligned}
& \int_{t_{0}}^{t_{N}} D\left(t_{0}, u\right) \cdot E_{Q^{t_{u}}}\left[P(u, K) \mid \mathbb{F}_{0}\right] \cdot \pi_{x}(u) d u \\
& +D\left(t_{0}, t_{N}\right) \cdot E_{Q^{t_{N}}}\left[P\left(t_{N}, K\right) \mid \mathbb{F}_{0}\right] \cdot\left(1-\int_{t_{0}}^{t_{N}} \pi_{x}(u) d u\right) \\
= & \sum_{i=0}^{N-1} K\left(t_{i}\right) \cdot D\left(t_{0}, t_{i}\right) \cdot\left(1-\int_{t_{0}}^{t_{i}} \pi_{x}(u) d u\right)=B_{1} .
\end{aligned}
$$


In the case of the $S P$-scheme the minimum payoff is equal to a convex combination of the portfolio value and the sum of the contributions compounded at the rate $g$. Therefore the additional part in this case equals the function $B_{2}(g)$ defined as

$$
\begin{aligned}
B_{2}(g):= & \int_{t_{0}}^{t_{N}} D\left(t_{0}, u\right) \cdot A(u, g) \cdot \pi_{x}(u) d u+D\left(t_{0}, t_{N}\right) \cdot A\left(t_{N}, g\right) \cdot\left(1-\int_{t_{0}}^{t_{N}} \pi_{x}(u) d u\right) \\
= & \int_{t_{0}}^{T}\left(\sum_{i=0}^{n^{*}(u)} K\left(t_{i}\right) \cdot D\left(t_{0}, u\right) \cdot \exp \left\{g \cdot\left(u-t_{i}\right)\right\}\right) \cdot \pi_{x}(u) d u \\
& +\left(\sum_{i=0}^{N-1} K\left(t_{i}\right) \cdot D\left(t_{0}, t_{N}\right) \cdot \exp \left\{g \cdot\left(t_{N}-t_{i}\right)\right\}\right) \cdot\left(1-\int_{t_{0}}^{t_{N}} \pi_{x}(u) d u\right) .
\end{aligned}
$$

As a function of the guaranteed rate of return $g$ the expected discounted payment $B_{2}(g)$ is nonnegative, strictly increasing and convex with

$$
\lim _{g \rightarrow-\infty} B_{2}(g)=0 \text { and } \lim _{g \rightarrow+\infty} B_{2}(g)=+\infty .
$$

Furthermore, for $g=g^{*}$ as defined in Proposition 4.1 we have $B_{2}\left(g^{*}\right)=B_{1}$. The remaining part of the expected discounted benefit is given by the put option with respect to the portfolio value. Depending on the pension scheme chosen, this part is either a function of the guaranteed rate of return $g$ only or of $g$ and the investment share $\alpha$, and this function is defined by

$$
\begin{aligned}
R(\alpha, g):= & \int_{t_{0}}^{t_{N}} D\left(t_{0}, u\right) \cdot E_{Q^{t_{u}}}\left[[A(u, g)-\alpha \cdot P(u, K)]^{+} \mid \mathbb{F}_{0}\right] \cdot \pi_{x}(u) d u \\
& +D\left(t_{0}, t_{N}\right) \cdot E_{Q^{t_{N}}}\left[\left[A\left(t_{N}, g\right)-\alpha \cdot P\left(t_{N}, K\right)\right]^{+} \mid \mathbb{F}_{0}\right] \cdot\left(1-\int_{t_{0}}^{t_{N}} \pi_{x}(u) d u\right) .
\end{aligned}
$$

In case of the $I G$ - or the $S P$-pension scheme, it is sufficient to consider $R(1, g)$. As a function of the guaranteed rate of return $g$ the expected discounted option payoff is non-negative, strictly increasing and convex with

$$
\lim _{g \rightarrow-\infty} R(1, g)=0 \quad \text { and } \quad \lim _{g \rightarrow+\infty} R(1, g)=+\infty .
$$

Furthermore the growth and curvature of the function $R(1, g)$ is bounded from above by the growth and curvature of the expected discounted payment $B_{2}(g)$, i.e

$$
\begin{aligned}
0<\frac{\partial R(1, g)}{\partial g} & \leq \frac{\partial B_{2}(g)}{\partial g} \\
0<\frac{\partial^{2} R(1, g)}{\partial g^{2}} & \leq \frac{\partial^{2} B_{2}(g)}{\partial g^{2}} .
\end{aligned}
$$


Finally, the application of the put-call-parity implies $\forall \alpha \geq 0$

$$
\begin{aligned}
0 \leq & R_{\text {call }}(\alpha, g) \\
:= & \int_{t_{0}}^{t_{N}} D\left(t_{0}, u\right) \cdot E_{Q^{t_{u}}}\left[[\alpha \cdot P(u, K)-A(u, g)]^{+} \mid \mathbb{F}_{0}\right] \cdot \pi_{x}(u) d u \\
& +D\left(t_{0}, t_{N}\right) \cdot E_{Q^{t_{N}}}\left[\left[\alpha \cdot P\left(t_{N}, K\right)-A\left(t_{N}, g\right)\right]^{+} \mid \mathbb{F}_{0}\right] \cdot\left(1-\int_{t_{0}}^{t_{N}} \pi_{x}(u) d u\right) \\
= & \int_{t_{0}}^{t_{N}} D\left(t_{0}, u\right) \cdot E_{Q^{t_{u}}}\left[[A(u, g)-\alpha \cdot P(u, K)]^{+} \mid \mathbb{F}_{0}\right] \cdot \pi_{x}(u) d u \\
& +D\left(t_{0}, t_{N}\right) \cdot E_{Q^{t_{N}}}\left[\left[A\left(t_{N}, g\right)-\alpha \cdot P\left(t_{N}, K\right)\right]^{+} \mid \mathbb{F}_{0}\right] \cdot\left(1-\int_{t_{0}}^{t_{N}} \pi_{x}(u) d u\right) \\
& +\alpha \cdot\left(\int_{t_{0}}^{t_{N}} D\left(t_{0}, u\right) \cdot E_{Q^{t_{u}}}\left[P(u, K) \mid \mathbb{F}_{0}\right] \cdot \pi_{x}(u) d u\right. \\
& \left.\quad+D\left(t_{0}, t_{N}\right) \cdot E_{Q^{t_{N}}}\left[P\left(t_{N}, K\right) \mid \mathbb{F}_{0}\right] \cdot\left(1-\int_{t_{0}}^{t_{N}} \pi_{x}(u) d u\right)\right) \\
& -\int_{t_{0}}^{t_{N}} D\left(t_{0}, u\right) \cdot A(u, g) \cdot \pi_{x}(u) d u+D\left(t_{0}, t_{N}\right) \cdot A\left(t_{N}, g\right) \cdot\left(1-\int_{t_{0}}^{t_{N}} \pi_{x}(u) d u\right) \\
= & R(\alpha, g)+\alpha \cdot B_{1}-B_{2}(g),
\end{aligned}
$$

which yields $\forall \alpha \geq 0$

$$
\begin{aligned}
R(\alpha, g) & \geq B_{2}(g)-\alpha \cdot B_{1} \\
\lim _{g \rightarrow+\infty} R(\alpha, g)+\alpha \cdot B_{1}-B_{2}(g) & =\lim _{g \rightarrow+\infty} R_{\text {call }}(\alpha, g)=0
\end{aligned}
$$

With this notation the functional relationship between the investment fraction $\alpha$ and the guaranteed rate of return $g$ on the set of admissible contracts can be expressed as follows:

- In the case of the $I G$-pension scheme the function of the investment share on the set of admissible contracts is uniquely determined by:

$$
\left(\alpha_{I G}(g), g\right) \in I_{x, I G}\left(K\left(t_{0}\right), \ldots, K\left(t_{N-1}\right)\right) \quad \Leftrightarrow \quad \alpha_{I G}(g):=\frac{B_{1}}{B_{1}+R(1, g)}
$$

- In the case of the $C G$-pension the functional relationship is implicitly given as the solution of the following non-linear problem

$$
\begin{aligned}
& \left(\alpha_{C G}(g), g\right) \in I_{x, C G}\left(K\left(t_{0}\right), \ldots, K\left(t_{N-1}\right)\right) \\
\Leftrightarrow \quad & \alpha_{C G}(g) \text { is a solution of } B_{1}=\alpha_{C G}(g) \cdot B_{1}+R\left(\alpha_{C G}(g), g\right)
\end{aligned}
$$

- In the case of the $S P$-pension scheme the function of the investment share on the set of admissible contracts is uniquely determined by:

$$
\begin{aligned}
& \left(\alpha_{S P}(g), g\right) \in I_{x, S P}\left(K\left(t_{0}\right), \ldots, K\left(t_{N-1}\right)\right) \\
\Leftrightarrow \quad & \alpha_{S P}(g):=\frac{B_{1}-B_{2}(g)}{B_{1}-B_{2}(g)+R(1, g)}
\end{aligned}
$$

For each pension scheme $c \in\{I G, C G, S P\}$ we can now characterize the set of admissible contracts

$$
I_{x, c}\left(K\left(t_{0}\right), \ldots, K\left(t_{N-1}\right)\right) \subset \mathbb{R}^{2} .
$$

The simplest situation is described by the $I G$-scheme. In this case the set of admissible contracts satisfies the following property 
Theorem 4.3 Let $\left(K\left(t_{0}\right), \ldots, K\left(t_{N-1}\right)\right)$ be a non-negative contribution sequence and consider an investment fraction $\alpha_{I G}(g)$ such that

$$
\left(\alpha_{I G}(g), g\right) \in I_{x, I G}\left(K\left(t_{0}\right), \ldots, K\left(t_{N-1}\right)\right) .
$$

Then $\alpha_{I G}(g)$ as a function of the guaranteed rate of return $g$ is non-negative and strictly decreasing with

$$
\lim _{g \rightarrow-\infty} \alpha_{I G}(g)=1 .
$$

Furthermore for $g \rightarrow+\infty$ the contract degenerates, i.e. the only admissible contract is determined by the contribution sequence equal to zero.

Proof: Consider first the situation of a non-negative contribution with positive present value, i.e. $K\left(t_{i}\right)>0$ for at least one investment date $t_{i} \in \underline{\underline{T}}$. In this case $B_{1}$ is greater than zero and the statement is a direct consequence of the functional form of the investment fraction as defined by equation (4.10) and the monotonicity and limit property for $g \rightarrow-\infty$ of $R(1, g)$ as given by (4.6). If instead $g \rightarrow+\infty$ the function $\alpha_{I G}(g)$ converges to zero. This implies that the only admissible pension scheme in this limit case is determined by the contribution sequence equal to zero.

By (4.12) the investment fraction of $S P$-pension scheme is explicitly determined and has the following properties:

Theorem 4.4 Let $\left(K\left(t_{0}\right), \ldots, K\left(t_{N-1}\right)\right)$ be a non-negative contribution sequence and consider an investment fraction $\alpha_{S P}(g)$ such that

$$
\left(\alpha_{S P}(g), g\right) \in I_{x, S P}\left(K\left(t_{0}\right), \ldots, K\left(t_{N-1}\right)\right)
$$

then $\alpha_{S P}(g)$ as a function of the guaranteed rate of return $g$ is non-negative, strictly decreasing and concave with

$$
\begin{aligned}
\alpha_{S P}\left(g^{*}\right) & =0, \\
\lim _{g \rightarrow-\infty} \alpha_{S P}(g) & =1, \\
\lim _{g \rightarrow+\infty} \alpha_{S P}(g) & =-\infty,
\end{aligned}
$$

where $g^{*}$ is defined as in Proposition 4.1.

Proof: By (4.12) the investment fraction is determined by

$$
\alpha_{S P}(g):=\frac{B_{1}-B_{2}(g)}{B_{1}-B_{2}(g)+R(1, g)}=\frac{B_{1}-B_{2}(g)}{R_{\text {Call }}(1, g)},
$$

where $R_{\text {Call }}(1, g)=B_{1}-B_{2}(g)+R(1, g)$. In particular (4.9) implies that the denominator is non-negative for all $g$. Furthermore $R(1, g)$ and $B_{2}(g)$ are non-negative and increasing functions of the guaranteed rate of return with $B_{2}(g)>B_{2}\left(g^{*}\right)=B_{1}>0$ for $g>g^{*}$. These properties imply $\alpha_{S P}\left(g^{*}\right)=0$ and furthermore the limit behavior of the investment share. Denote by $R^{\prime}(1, g), R^{\prime \prime}(1, g), B_{2}^{\prime}(g)$ and $B_{2}^{\prime \prime}(g)$ the first and second derivative with respect to the guaranteed rate of return. After some simplification the first derivative of the investment fraction as a function of $g$ can be computed as

$$
\frac{\partial \alpha_{S P}(g)}{\partial g}=-\frac{R(1, g) \cdot B_{2}^{\prime}(g)+\left(B_{1}-B_{2}(g)\right) \cdot R^{\prime}(1, g)}{R_{\text {Call }}^{2}(1, g)}<-\frac{R_{\text {Call }}(1, g) \cdot R^{\prime}(1, g)}{R_{\text {Call }}^{2}(1, g)}<0,
$$

where the first inequality is an implication of equation (4.7) and the second of equation (4.9). Again by straightforward differentiation the second derivative of the investment share as a function of the guaranteed rate of return is given by

$$
\begin{aligned}
\frac{\partial^{2} \alpha_{S P}(g)}{\partial g^{2}}= & -\frac{R(1, g) \cdot B_{2}^{\prime \prime}(g)+\left(\left(B_{1}-B_{2}(g)\right) \cdot R^{\prime \prime}(1, g)\right.}{R_{\text {Call }}^{2}(1, g)} \\
& +2 \cdot \frac{\left(R(1, g) \cdot B_{2}^{\prime}(g)+\left(B_{1}-B_{2}(g)\right) \cdot R^{\prime}(1, g)\right) \cdot\left(R^{\prime}(1, g)-B_{2}^{\prime}(g)\right)}{R_{\text {Call }}^{3}(1, g)} .
\end{aligned}
$$


The first term is non-positive since $B_{2}^{\prime \prime}(g) \geq R^{\prime \prime}(1, g)$ and $B_{1}-B_{2}(g)+R(1, g)=R_{\text {call }}(1, g) \geq 0$. The second term is also non-positive since $B_{2}^{\prime}(g) \geq R^{\prime}(1, g)$. Therefore the second derivative of the investment fraction is non-positive which implies the concavity as a function of the guaranteed rate of return.

The analysis of the $C G$-scheme is a little more complicated since the investment fraction is determined as the solution of a non-linear equation given by (4.11).

Theorem 4.5 Let $\left(K\left(t_{0}\right), \ldots, K\left(t_{N-1}\right)\right)$ be a non-negative contribution sequence and consider an investment fraction $\alpha_{C G}(g)$ such that

$$
\left(\alpha_{C G}(g), g\right) \in I_{x, C G}\left(K\left(t_{0}\right), \ldots, K\left(t_{N-1}\right)\right),
$$

and let $g^{*}$ be the critical guaranteed rate of return defined in Proposition 4.1.

- There exist no admissible CG-pension contracts with a guaranteed rate of return $g>g^{*}$.

- For $g \leq g^{*}$ the investment fraction $\alpha_{C G}(g)$ as a function of the guaranteed rate of return $g$ is non-negative, strictly decreasing and concave with

$$
\begin{aligned}
\alpha_{P S}\left(g^{*}\right) & =0, \\
\lim _{g \rightarrow-\infty} \alpha_{P S}(g) & =1 .
\end{aligned}
$$

Proof: The investment fraction is characterized as the solution of equation (4.11), i.e.

$$
B_{1}=\alpha_{C G} \cdot B_{1}+R\left(\alpha_{C G}, g\right)=B_{2}(g)+R_{C a l l}\left(\alpha_{C G}, g\right),
$$

where $R_{\text {Call }}(\alpha, g)$ is defined in (4.9) as $R_{\text {Call }}(\alpha, g):=\alpha \cdot B_{1}-B_{2}(g)+R(\alpha, g)$. Define the right hand side of (4.13) as a function $f(\alpha, g)$. Since $B_{2}(g)$ is strictly increasing in $g$ with $B_{2}\left(g^{*}\right)=B_{1}>0$ the investment fraction $\alpha^{*}:=\alpha_{C G}\left(g^{*}\right)$ must be a solution of

$$
B_{1}=f\left(\alpha^{*}, g^{*}\right)=\alpha^{*} \cdot B_{1}+R\left(\alpha^{*}, g\right)=B_{2}\left(g^{*}\right)+R_{\text {Call }}\left(\alpha^{*}, g\right) \Leftrightarrow 0=R_{\text {Call }}\left(\alpha^{*}, g\right),
$$

which implies $\alpha^{*}=0$. Furthermore since $R_{\text {Call }}(\alpha, g) \forall g$ is non-negative and $B_{2}(g)>B_{2}\left(g^{*}\right)$ for $g>g^{*}$, there exists no admissible contract for $g>g^{*}$. The limit result is a consequence of

$$
\lim _{g \rightarrow-\infty} f(\alpha, g)=\lim _{g \rightarrow-\infty}\left(R(\alpha, g)+\alpha \cdot B_{1}\right)=\alpha B_{1},
$$

which in conjunction with (4.13) implies that for $g \rightarrow-\infty$ the investment share of an admissible contract must converge to one. The function $f(\alpha, g)$ is twice differentiable in both parameters with $f_{\alpha}, f_{g}, f_{\alpha, \alpha}, f_{g, g}>0$ and cross derivative $f_{\alpha, g}<0$. The solution $\alpha(g)$ such that $c=f(\alpha, g)$ must satisfy

$$
0=f_{\alpha} d \alpha+f_{g} d g \Leftrightarrow \frac{d \alpha}{d g}=-\frac{f_{g}}{f_{\alpha}}<0
$$

Which implies that the solution $\alpha(g)$ is decreasing in the guaranteed rate of return $g$. Furthermore the second total differential implies

$$
\begin{aligned}
0 & =f_{\alpha, \alpha}(d \alpha)^{2}+2 \cdot f_{\alpha, g} d \alpha d g+f_{g, g}(d g)^{2} \\
\Leftrightarrow \frac{d \alpha^{2}}{d g^{2}} & =-\frac{f_{g, g}}{f_{\alpha, \alpha}}-2 \cdot \frac{f_{\alpha, g}}{f_{\alpha, \alpha}} \cdot \frac{d \alpha}{d g}<0
\end{aligned}
$$

since $f(\alpha, g)$ is convex in both parameters and the cross derivative is negative. as a consequence the investment share is a concave function of the guaranteed rate of return.

In the case of an investment contract, i.e. without the termination uncertainty of the pension situation, the investment fractions of all three schemes are ordered in a monotonic fashion (see Proposition 2.1). The same property holds in case of a pension contract with the possibility of early termination. 
Proposition 4.6 Consider a non-negative contribution sequence $\left(K\left(t_{0}\right), \ldots, K\left(t_{N-1}\right)\right)$ with positive present value and define $g^{*}$ as in Proposition 4.1. For all guaranteed rates of return $g \leq g^{*}$ such that

$$
\begin{aligned}
\left(\alpha_{I G}(g), g\right) & \in I_{x, I G}\left(K\left(t_{0}\right), \ldots, K\left(t_{N-1}\right)\right) \\
\left(\alpha_{C G}(g), g\right) & \in I_{x, C G}\left(K\left(t_{0}\right), \ldots, K\left(t_{N-1}\right)\right) \\
\left(\alpha_{S P}(g), g\right) & \in I_{x, S P}\left(K\left(t_{0}\right), \ldots, K\left(t_{N-1}\right)\right)
\end{aligned}
$$

the investment fractions of the admissible contracts are ordered by

$$
\alpha_{I G}(g) \geq \alpha_{C G}(g) \geq \alpha_{S P}(g) \quad \forall g \leq g^{*} .
$$

Proof: Let $g \leq g^{*}$ be an arbitrary guaranteed rate of return and consider

$$
\left(\alpha_{C G}(g), g\right) \in I_{x, C G}\left(K\left(t_{0}\right), \ldots, K\left(t_{N-1}\right)\right) .
$$

The investment fraction $\alpha_{C G}(g)$ is therefore a solution of

$$
B_{1}=\alpha_{C G}(g) \cdot B_{1}+R\left(\alpha_{C G}(g), g\right) .
$$

For $\alpha \in[0,1]$ the expected discounted option term $R(\alpha, g)$ is decreasing, with $R(\alpha, g) \geq R(1, g)$. Therefore the right hand side of the above equation is bounded from below by

$$
\begin{aligned}
B_{1} & =\alpha_{C G}(g) \cdot B_{1}+R\left(\alpha_{C G}(g), g\right) \geq \alpha_{C G}(g) \cdot B_{1}+R(1, g) \\
\Leftrightarrow \alpha_{C G}(g) & \leq \frac{B_{1}}{B_{1}+R(1, g)}=\alpha_{I G}(g) \quad \forall g \leq g^{*} .
\end{aligned}
$$

To derive the upper bound consider again the put-call-parity relation $R_{\text {Call }}(\alpha, g)=B_{1}-B_{2}(g)+$ $R(\alpha, g)$ as in equation (4.9). This implies that the expected discounted option part is bounded from above for $\alpha \in[0,1]$

$$
\begin{aligned}
B_{1} & =\alpha_{C G}(g) \cdot B_{1}+R\left(\alpha_{C G}(g), g\right) \\
& =\alpha_{C G}(g) \cdot B_{1}+B_{2}(g)-\alpha_{C G} \cdot B_{1}+R_{\text {Call }}\left(\alpha_{C G}, g\right) \\
& =B_{2}(g)+R_{\text {Call }}\left(\alpha_{C G}, g\right) \\
& \leq B_{2}(g)+\alpha_{C G} \cdot R_{\text {Call }}(1, g) \\
& =B_{2}(g)+\alpha_{C G} \cdot\left(B_{1}-B_{2}(g)+R(1, g)\right) \\
\Leftrightarrow \alpha_{C G}(g) & \geq \frac{B_{1}-B_{2}(g)}{B_{1}-B_{2}(g)+R(1, g)}=\alpha_{S P}(g) \quad \forall g \leq g^{*} .
\end{aligned}
$$

\section{$5 \quad$ Valuation of the Embedded Average Return Options}

The computation of the set of admissible contracts $(\alpha, g) \in I_{x, c}\left(K\left(t_{0}\right), \ldots, K\left(t_{N-1}\right)\right)$ for $c \in$ $\{I G, C G, S P\}$ involves the computation of a sequence of option contracts which are related to Asian-type put options. To evaluate these options, we will determine their upper and lower bounds, extending the conditional technique independently introduced by Curran (1994) and Rogers and Shi (1995). In contrast to these contributions, we also include interest rate risk. The put options in our case are defined on the discrete average return instead of the average funds value. Therefore the contract situation as well as the model assumptions differ from those investigated by Curran (1994) and Rogers and Shi (1995). The lower bound of an Asian type put option is given by Curran as well as by Rogers and Shi in the form of an integral equation which is solved by numerical integration. Applying arguments from Nielsen and Sandmann (2002), we derive a closed form solution of the 
resulting integral equation for the lower bound and determine a closed form approximation of the maximum error which is smaller than the one derived by Curran, Rogers and Shi.

To start the analysis we assume a general Gaussian framework of the financial market defined by the stochastic processes of the underlying stock index and the term structure of interest rates, with deterministic volatility functions. Thus the dynamics of the underlying stock index under the spot risk-neutral measure ${ }^{5} Q^{\beta}$ are given by

$$
\frac{d S(t)}{S(t)}=r(t) d t+\sigma_{S}(t) d W_{\beta}(t)
$$

where $r(t)$ is the continuously compounded short rate of interest, $W_{\beta}(t)$ is a $d$-dimensional vectorvalued Brownian motion, $\sigma_{S}(t)$ is a deterministic, $d$-dimensional vector-valued function of $t$ and $\sigma_{S}(t) d W_{\beta}(t)$ is to be understood as a sum product.

The interest rate dynamics are given by a Gauss/Markov Heath, Jarrow and Morton (1992) model, i.e. the instantaneous forward rates satisfy

$$
\begin{aligned}
d f(t, T) & =\psi(t, T) \psi^{*}(t, T) d t+\psi(t, T) d W_{\beta}(t) \\
\psi^{*}(t, T) & =\int_{t}^{T} \psi(t, u) d u
\end{aligned}
$$

with $\psi(\cdot, \cdot)$ a deterministic, $d$-dimensional vector-valued function with components

$$
\psi_{i}(t, T)=\sigma_{i}(t) e^{-a_{i}(T-t)}
$$

It follows that the zero coupon bond price dynamics are

$$
d D(t, T)=D(t, T)\left(r(t) d t-\psi^{*}(t, T) d W_{\beta}(t)\right)
$$

For simplicity of the exposition define $n:=n^{*}(\tau)$ for a fixed time $\left.\left.\left.\left.\tau \in\right] t_{n}, t_{n+1}\right] \subseteq\right] t_{0}, t_{N}\right]$. If the exercise of the pension contract occurs at time $\tau$ the option part of the payoff for all three schemes is related to

$$
[A(\tau, g)-\gamma \cdot P(\tau, K)]^{+}=\left[\sum_{i=0}^{n} K\left(t_{i}\right) \cdot \exp \left\{g \cdot\left(\tau-t_{i}\right)\right\}-\gamma \cdot \sum_{i=0}^{n} K\left(t_{i}\right) \cdot \frac{S(\tau)}{S\left(t_{i}\right)}\right]^{+}
$$

where $\gamma$ is either equal to 1 or $\alpha$. The arbitrage-free price at time $t_{0}$ of this average return Asian-type option is given by

$$
\operatorname{Put}\left(t_{0}, \tau, \gamma, A(\tau, g)\right):=D\left(t_{0}, t_{j}\right) \cdot E_{Q^{\tau}}\left[\left[A(\tau, g)-\gamma \cdot \sum_{i=0}^{n} K\left(t_{i}\right) \cdot \frac{S(\tau)}{S\left(t_{i}\right)}\right]^{+}\right],
$$

with $\left.\left.\left.\tau \in] t_{n}, t_{n+1}\right] \subseteq\right] t_{0}, t_{N}\right]$.

Consider now the solution of the stochastic differential equation for the quotient $S(\tau) / S\left(t_{i}\right)$ under the time $\tau$ forward measure $Q^{\tau}$ (i.e. the equivalent martingale measure associated with taking the zero coupon bond $D(t, \tau)$ as the numeraire). By definition of $Q^{\tau}$ we have

$$
S(\tau)=\frac{S\left(t_{0}\right)}{D\left(t_{0}, \tau\right)} \exp \left\{\int_{t_{0}}^{\tau}\left(\sigma_{S}(u)+\psi^{*}(u, \tau)\right) d W_{\tau}(u)-\frac{1}{2} \int_{t_{0}}^{\tau}\left(\sigma_{S}(u)+\psi^{*}(u, \tau)\right)^{2} d u\right\}
$$

\footnotetext{
${ }^{5}$ The spot risk-neutral measure is the equivalent martingale measure associated with taking the continuously compounded savings account

$$
\beta(t)=\exp \left\{\int_{t_{0}}^{t} r(s) d s\right\}
$$
}

as the numeraire. 
Furthermore, under the time $t_{i}$ forward measure $Q^{t_{i}}$,

$$
S\left(t_{i}\right)=\frac{S\left(t_{0}\right)}{D\left(t_{0}, t_{i}\right)} \exp \left\{\int_{t_{0}}^{t_{i}}\left(\sigma_{S}(u)+\psi^{*}\left(u, t_{i}\right)\right) d W_{t_{i}}(u)-\frac{1}{2} \int_{t_{0}}^{t_{i}}\left(\sigma_{S}(u)+\psi^{*}\left(u, t_{i}\right)\right)^{2} d u\right\}
$$

and by well-known change-of-numeraire results ${ }^{6}$

$$
d W_{t_{i}}(u)=d W_{\tau}(u)+\left(\psi^{*}\left(u, t_{i}\right)-\psi^{*}(u, \tau)\right) d u
$$

Thus the solution of the stochastic process $\left(\frac{S(\tau)}{S\left(t_{i}\right)}\right)$ for $i=0, \ldots, n$ is equal to

$$
\frac{S(\tau)}{S\left(t_{i}\right)}=H\left(t_{0}, t_{i}, \tau\right) \cdot \exp \left\{Z\left(t_{0}, t_{i}, \tau\right)\right\}
$$

where $H\left(t_{0}, t_{i}, \tau\right)$ is a deterministic function defined by

$$
\begin{aligned}
& H\left(t_{0}, t_{i}, \tau\right) \\
& :=\frac{D\left(t_{0}, t_{i}\right)}{D\left(t_{0}, \tau\right)} \quad \exp \left\{-\frac{1}{2} \int_{t_{0}}^{t_{i}}\left(\psi^{*}\left(u, t_{i}\right)-\psi^{*}(u, \tau)\right)^{2} d u-\frac{1}{2} \int_{t_{i}}^{\tau}\left(\sigma_{S}(u)+\psi^{*}(u, \tau)\right)^{2} d u\right\}
\end{aligned}
$$

and $Z\left(t_{0}, t_{i}, \tau\right)$ is a normally distributed random variable with expectation equal to zero under $Q^{\tau}$. $Z\left(t_{0}, t_{i}, \tau\right)$ is determined by

$$
\begin{aligned}
Z\left(t_{0}, t_{i}, \tau\right) & \int_{t_{i}}^{\tau} \sigma_{S}(u) d W_{\tau}(u)+\int_{t_{0}}^{\tau} \psi^{*}(u, \tau) d W_{\tau}(u)-\int_{t_{0}}^{t_{i}} \psi^{*}\left(u, t_{i}\right) d W_{\tau}(u) .
\end{aligned}
$$

Since the sum of the random variables, $Z\left(t_{0}, t_{i}, \tau\right)$ is normally distributed, a standardized normal distributed variable $Z_{\tau}$ is defined by

$$
Z_{\tau}:=\frac{1}{\Omega_{\tau}} \sum_{i=0}^{n} Z\left(t_{0}, t_{i}, \tau\right),
$$

where $\Omega_{\tau}$ is determined such that $\operatorname{Var}_{Q^{\tau}}\left[Z_{\tau}\right]=1$. Under the conditional approach the lower bound of the Asian type average return put option (5.1) is established through Jensen's inequality as

$$
\begin{aligned}
& D\left(t_{0}, \tau\right) \cdot E_{Q^{\tau}}\left[E_{Q^{\tau}}\left[\left[A(\tau, g)-\gamma \cdot \sum_{i=0}^{n} K\left(t_{i}\right) \cdot \frac{S(\tau)}{S\left(t_{i}\right)}\right]^{+} \mid Z_{\tau}\right]\right] \\
\geq & D\left(t_{0}, \tau\right) \cdot E_{Q^{\tau}}\left[E_{Q^{\tau}}\left[A(\tau, g)-\gamma \cdot \sum_{i=0}^{n} K\left(t_{i}\right) \cdot \frac{S(\tau)}{S\left(t_{i}\right)} \mid Z_{\tau}\right]^{+}\right] \\
= & : \quad \operatorname{Put}^{l}\left(t_{0}, \tau, \gamma, A(\tau, g)\right) .
\end{aligned}
$$

As a result of Jensen's inequality and the Gaussian structure the inner conditional expectation can be computed and is equal to

$$
\begin{aligned}
& E_{Q^{\tau}}\left[A(\tau, g)-\gamma \cdot \sum_{i=0}^{n} K\left(t_{i}\right) \cdot \frac{S(\tau)}{S\left(t_{i}\right)} \mid Z_{\tau}=z\right] \\
= & A(\tau, g)-\gamma \cdot \sum_{i=0}^{n} K\left(t_{i}\right) \cdot H\left(t_{0}, t_{i}, \tau\right) \cdot \exp \left\{m_{\tau}\left(t_{i}\right) \cdot z+\frac{1}{2} \cdot v_{\tau}^{2}\left(t_{i}, t_{i}\right)\right\},
\end{aligned}
$$

\footnotetext{
${ }^{6}$ See e.g. Geman, El Karoui and Rochet (1995).
} 
where

$$
\begin{aligned}
m_{\tau}\left(t_{i}\right) & :=E_{Q^{\tau}}\left[Z_{\tau} \cdot Z\left(t_{0}, t_{i}, \tau\right)\right] \\
\nu_{\tau}^{2}\left(t_{i}, t_{k}\right) & :=\operatorname{cov}_{Q^{\tau}}\left[Z\left(t_{0}, t_{i}, \tau\right), Z\left(t_{0}, t_{k}, \tau\right) \mid Z_{\tau}\right] .
\end{aligned}
$$

The solution of the conditional expectation (5.7) can be rewritten as in terms of a sum of convex functions

$$
\begin{aligned}
f_{i}(z) & :=K\left(t_{i}\right) \cdot H\left(t_{0}, t_{i}, t_{j}\right) \cdot \exp \left\{m_{\tau}\left(t_{i}\right) \cdot z+\frac{1}{2} \cdot \nu_{\tau}^{2}\left(t_{i}, t_{i}\right)\right\} \\
& =K\left(t_{i}\right) \cdot \frac{D\left(t_{0}, t_{i}\right)}{D\left(t_{0}, \tau\right)} \cdot \exp \left\{m_{M, j}\left(t_{i}\right) \cdot z-\frac{1}{2} \cdot m_{\tau}^{2}\left(t_{i}\right)\right\} .
\end{aligned}
$$

As a consequence the equation $A(\tau, g)-\gamma \cdot \sum_{i=0}^{M} f_{i}(z)=0$ has infinitely many, zero, one or two solutions which we may define as follows

\section{Definition 4}

- If $A(\tau, g)-\sum_{i=0}^{n} f_{i}(z)<0 \quad \forall z$, define $z^{*}=z^{* *}:=0$,

- if $A(\tau, g)-\sum_{i=0}^{n} f_{i}(z) \geq 0 \quad \forall z$, which could only be the case if $m_{\tau}\left(t_{i}\right)=0 \quad \forall i$, we define $z^{*}:=-\infty$ and $z^{* *}:=\infty$,

- if $A(\tau, g)-\sum_{i=0}^{n} f_{i}(z)=0$ has one solution and $m_{\tau}\left(t_{i}\right) \nless 0 \quad \forall i$, we define $z^{*}:=-\infty$ and denote the solution by $z^{* *}$,

- if $A(\tau, g)-\sum_{i=0}^{n} f_{i}(z)=0$ has one solution and $m_{\tau}\left(t_{i}\right) \ngtr 0 \quad \forall i$, we denote the solution by $z^{*}$ and define $z^{* *}:=\infty$,

- if $A(\tau, g)-\sum_{i=0}^{n} f_{i}(z)=0$ has two solutions, we denote these by $z^{*}$ and $z^{* *}$ and let $z^{*}<z^{* *}$.

With this notation and applying the same arguments as in Nielsen and Sandmann (2002) the lower bound of the conditional approach is equal to

$$
\begin{aligned}
& \operatorname{Put}^{l}\left(t_{0}, \tau, \gamma, A(\tau, g)\right) \\
= & D\left(t_{0}, \tau\right) \cdot\left[A(\tau, g) \cdot E_{Q^{\tau}}\left[1_{\left\{z \geq z^{*}\right\}}\right]-\gamma \cdot \sum_{i=0}^{n} E_{Q^{\tau}}\left[f_{i}(z) 1_{\left\{z \geq z^{*}\right\}}\right]\right. \\
& -A(\tau, g) \cdot E_{Q^{\tau}}\left[1_{\left\{z \geq z^{* *}\right\}}\right]+\gamma \cdot \sum_{i=0}^{n} E_{Q^{\tau}}\left[\left(f_{i}(z) 1_{\left\{z \geq z^{* *}\right\}}\right]\right] \\
= & {\left[D\left(t_{0}, \tau\right) \cdot A(\tau, g) \cdot \Phi\left(-z^{*}\right)-\gamma \cdot \sum_{i=0}^{n} D\left(t_{0}, t_{i}\right) \cdot \Phi\left(-z^{*}+m_{\tau}\left(t_{i}\right)\right)\right.} \\
& \left.-A(\tau, g) \cdot D\left(t_{0}, \tau\right) \cdot \Phi\left(-z^{* *}\right)+\gamma \cdot \sum_{i=0}^{n} D\left(t_{0}, t_{i}\right) \cdot \Phi\left(-z^{* *}+m_{\tau}\left(t_{i}\right)\right)\right],
\end{aligned}
$$

where $\Phi(\cdot)$ denotes the cumulative standard normal distribution.

If $\varepsilon\left(t_{0}, \tau, \gamma, A(\tau, g)\right)$ is an upper bound to the error made by applying the conditioning method, then an upper bound for the value of the average return put option is defined by

$$
\operatorname{Put}^{u}\left(t_{0}, \tau, \gamma, A(\tau, g)\right):=\operatorname{Put}^{l}\left(t_{0}, \tau, \gamma, A(\tau, g)\right)+\varepsilon\left(t_{0}, \tau, \gamma, A(\tau, g)\right) .
$$

The construction of an upper bound for this error is now based on the following observation. The conditioning by $Z_{\tau}$ is equivalent to the conditioning by the geometric average since the conditioning 
variable can be rewritten as

$$
\begin{aligned}
Z_{\tau} & =\frac{\ln (G(\tau))-E_{Q^{\tau}}[\ln (G(\tau))]}{\left(\operatorname{Var}_{Q^{\tau}}[\ln (G(\tau))]\right)^{\frac{1}{2}}}, \\
G_{\tau} & :=\left(\prod_{i=0}^{n} K\left(t_{i}\right) \cdot \frac{S(\tau)}{S\left(t_{i}\right)}\right)^{\frac{1}{n+1}} .
\end{aligned}
$$

Furthermore the arithmetic average is bounded from below by the the geometric average, which implies that

$$
G(\tau) \leq \frac{1}{n+1} \cdot \sum_{i=0}^{n} K\left(t_{i}\right) \cdot \frac{S(\tau)}{S\left(t_{i}\right)}
$$

As a consequence the error between the lower bound and the true value of the average return put-option is restricted to the set

$$
\left\{G_{\tau} \leq \frac{A(\tau, g)}{\gamma \cdot(n+1)}\right\}=\left\{Z_{\tau} \leq d\right\}
$$

where $d$ is given by

$$
d:=\frac{\ln \left(\frac{A(\tau, g)}{\gamma \cdot(n+1)}\right)-E_{Q^{\tau}}\left[\ln \left(G_{\tau}\right)\right]}{\left(\operatorname{Var}_{Q^{\tau}}\left[\ln \left(G_{\tau}\right)\right]\right)^{\frac{1}{2}}}=\frac{n+1}{\Omega_{\tau}} \cdot \ln \left[\frac{A(\tau, g)}{\gamma \cdot(n+1) \cdot\left(\prod_{i=0}^{n} K\left(t_{i}\right) \cdot H\left(t_{0}, t_{i}, \tau\right)\right)^{\frac{1}{n+1}}}\right] .
$$

Denoting by $\phi(\cdot)$ the standard normal density function for $Z_{\tau}$ a bound can therefore be expressed as

$$
\begin{aligned}
0 \leq & E_{Q^{\tau}}\left[E_{Q^{\tau}}\left[\left[A(\tau, g)-\gamma \cdot \sum_{i=0}^{n} K\left(t_{i}\right) \cdot H\left(t_{0}, t_{i}, \tau\right) \cdot \exp \left\{Z\left(t_{0}, t_{i}, \tau\right)\right\}\right]^{+} \mid Z_{\tau}\right]\right. \\
& \left.-\left[E_{Q^{\tau}}\left[A(\tau, g)-\gamma \cdot \sum_{i=0}^{n} K\left(t_{i}\right) \cdot H\left(t_{0}, t_{i}, \tau\right) \cdot \exp \left\{Z\left(t_{0}, t_{i}, \tau\right)\right\} \mid Z_{\tau}\right]\right]^{+}\right] \\
= & \int_{-\infty}^{d}\left(E_{Q^{\tau}}\left[\left[A(\tau, g)-\gamma \cdot \sum_{i=0}^{n} K\left(t_{i}\right) \cdot H\left(t_{0}, t_{i}, \tau\right) \cdot \exp \left\{Z\left(t_{0}, t_{i}, \tau\right)\right\}\right]^{+} \mid Z_{\tau}\right]\right. \\
& \left.-E_{Q^{\tau}}\left[A(\tau, g)-\gamma \cdot \sum_{i=0}^{n} K\left(t_{i}\right) \cdot H\left(t_{0}, t_{i}, \tau\right) \cdot \exp \left\{Z\left(t_{0}, t_{i}, \tau\right)\right\} \mid Z_{\tau}\right]^{+}\right) \phi(z) d z \\
\leq & \frac{1}{2} \int_{-\infty}^{d}\left(\operatorname{Var}_{Q^{\tau}}\left[\sum_{i=0}^{n} K\left(t_{i}\right) \cdot H\left(t_{0}, t_{i}, \tau\right) \cdot \exp \left\{Z\left(t_{0}, t_{i}, \tau\right)\right\} \mid Z_{\tau}\right]\right)^{\frac{1}{2}} \phi(z) d z \\
= & \frac{1}{2} \cdot E_{Q^{\tau}}\left[\left(\operatorname{Var}_{Q^{\tau}}\left[\sum_{i=0}^{n} K\left(t_{i}\right) \cdot H\left(t_{0}, t_{i}, \tau\right) \cdot \exp \left\{Z\left(t_{0}, t_{i}, \tau\right)\right\} \mid Z_{\tau}\right] 1_{\left\{Z_{\tau}<d\right\}}\right)^{\frac{1}{2}}\left(1_{\left\{Z_{\tau}<d\right\}}\right)^{\frac{1}{2}}\right] \\
\leq & \frac{1}{2} \cdot\left(E_{Q^{\tau}}\left[\operatorname{Var}_{Q^{\tau}}\left[\sum_{i=0}^{M} K\left(t_{i}\right) \cdot H\left(t_{0}, t_{i}, \tau\right) \cdot \exp \left\{Z\left(t_{0}, t_{i}, \tau\right)\right\} \mid Z_{\tau}\right] 1_{\left\{Z_{\tau}<d\right\}}\right]\right)^{\frac{1}{2}} \\
& \left(E_{Q^{\tau}}\left[1_{\left\{Z_{\tau}<d\right\}}\right]\right)^{\frac{1}{2}},
\end{aligned}
$$

where Hölder's inequality has been applied in the last inequality. The bound on the forward ${ }^{7}$ pricing

\footnotetext{
${ }^{7}$ To calculate the bound on the error in the present value of the option, this must be discounted by multiplying by $D\left(t_{0}, \tau\right)$.
} 
error of the conditional approach is therefore given by

$$
\begin{aligned}
& \varepsilon\left(t_{0}, \tau, \gamma, A(\tau, g)\right)=\frac{1}{2} \cdot \Phi(d)^{\frac{1}{2}} \\
& \cdot\left(E_{Q^{\tau}}\left[\operatorname{Var}_{Q^{\tau}}\left[\sum_{i=0}^{M} K\left(t_{i}\right) \cdot H\left(t_{0}, t_{i}, \tau\right) \cdot \exp \left\{Z\left(t_{0}, t_{i}, \tau\right)\right\} \mid Z_{\tau}\right] 1_{\left\{Z_{\tau}<d\right\}}\right]\right)^{\frac{1}{2}}
\end{aligned}
$$

To compute this, consider first the conditional variance term inside the expectation. To shorten notation, define

$$
\begin{aligned}
q_{i} & :=K\left(t_{i}\right) H\left(t_{0}, t_{i}, \tau\right) \\
Z_{i} & :=Z\left(t_{0}, t_{i}, \tau\right)
\end{aligned}
$$

Then we can write

$$
\begin{aligned}
& \operatorname{Var}_{Q^{\tau}}\left[\sum_{i=0}^{n} q_{i} e^{Z_{i}} \mid Z_{\tau}\right] \\
= & \sum_{k=1}^{n}\left(\operatorname{Var}_{Q^{\tau}}\left[q_{k} e^{Z_{k}} \mid Z_{\tau}\right]+2 \operatorname{Cov}_{Q^{\tau}}\left[q_{k} e^{Z_{k}}, \sum_{i=0}^{k-1} q_{i} e^{Z_{i}} \mid Z_{\tau}\right]\right)+\operatorname{Var}_{Q^{\tau}}\left[q_{0} e^{Z_{0}} \mid Z_{\tau}\right] \\
= & \sum_{k=0}^{n} \operatorname{Var}_{Q^{\tau}}\left[q_{k} e^{Z_{k}} \mid Z_{\tau}\right]+2 \sum_{k=1}^{n}\left(E_{Q^{\tau}}\left[q_{k} e^{Z_{k}} \sum_{i=0}^{k-1} q_{i} e^{Z_{i}} \mid Z_{\tau}\right]-E_{Q^{\tau}}\left[q_{k} e^{Z_{k}} \mid Z_{\tau}\right] \sum_{i=0}^{k-1} E_{Q^{\tau}}\left[q_{i} e^{Z_{i}} \mid Z_{\tau}\right]\right)
\end{aligned}
$$

Note that $Z_{k}$ given $Z_{\tau}$ is normally distributed with mean $Z_{\tau} \theta_{k}$,

$$
\theta_{k}:=\sqrt{\operatorname{Var}\left[Z\left(t_{0}, t_{k}, \tau\right)\right]} \operatorname{Corr}\left[Z\left(t_{0}, t_{k}, \tau\right), Z_{\tau}\right]
$$

and variance

$$
\sigma_{k}^{2}:=\left(1-\operatorname{Corr}\left[Z\left(t_{0}, t_{k}, \tau\right), Z_{\tau}\right]^{2}\right) \operatorname{Var}\left[Z\left(t_{0}, t_{k}, \tau\right)\right]
$$

Therefore

$$
\begin{aligned}
\operatorname{Var}_{Q^{\tau}}\left[q_{k} e^{Z_{k}} \mid Z_{\tau}\right] & =q_{k}^{2}\left(e^{\sigma_{k}^{2}}-1\right) e^{2 \theta_{k} Z_{\tau}+\sigma_{k}^{2}} \\
E_{Q^{\tau}}\left[q_{k} e^{Z_{k}} \mid Z_{\tau}\right] & =q_{k} e^{\theta_{k} Z_{\tau}+\frac{1}{2} \sigma_{k}^{2}}
\end{aligned}
$$

Similarly, $Z_{k}+Z_{i}$ given $Z_{\tau}$ is normally distributed with mean $Z_{\tau} \theta_{i, k}$,

$$
\theta_{i, k}:=\sqrt{\operatorname{Var}\left[Z_{k}+Z_{i}\right]} \operatorname{Corr}\left[Z_{k}+Z_{i}, Z_{\tau}\right]
$$

and variance

$$
\sigma_{i, k}^{2}:=\left(1-\operatorname{Corr}\left[Z_{k}+Z_{i}, Z_{\tau}\right]\right)^{2} \operatorname{Var}\left[Z_{k}+Z_{i}\right]
$$

Therefore

$$
E_{Q^{\tau}}\left[q_{k} e^{Z_{k}} q_{i} e^{Z_{i}} \mid Z_{\tau}\right]=q_{k} q_{i} e^{\theta_{i, k} Z_{\tau}+\frac{1}{2} \sigma_{i, k}^{2}}
$$

Thus the right hand side of (5.11) is a sum of terms of the type $c e^{a Z_{\tau}+b}$ and we can calculate the unconditional expectation in (5.10) as a sum of terms of the type

$$
E_{Q^{\tau}}\left[c e^{a Z_{\tau}+b} 1_{\left\{Z_{\tau}<d\right\}}\right]=c e^{\frac{1}{2} a^{2}+b} \Phi(d-a)
$$




\section{Numerical Results and Conclusion}

For the numerical analysis we have to specify the exercise distribution, the volatility functions of the risky fund and the term structure of interest rates and as well as the contract situations of the pension contracts. The numerical approximation of the Asian type average return put options is valid for any deterministic specification of the volatility functions.

For our numerical examples, we specify the stock index volatility as

$$
\sigma_{S}(t)=\left(\begin{array}{c}
0 \\
0.25
\end{array}\right) \quad \forall t
$$

and the interest rate volatility function as

$$
\psi(t, T)=\left(\begin{array}{c}
0.15 e^{-0.25(T-t)} \\
0
\end{array}\right) \quad \forall t
$$

i.e. the instantaneous correlation between the two processes is set to zero. In addition, we assume that the initial term structure is flat with an interest rate equal to $4 \%$ continuously compounded. As discussed earlier, the distribution of contract termination times is closely related to the mortality distribution. In fact one way to define the relationship between the termination distribution and the mortality distribution is by using a Wang transformation. Another possibility to take care of the difference between the termination and the mortality distribution is to adjust the mortality table by increasing the age of the investor. As an example we use a mortality table adjusted with the Makeham formula

$$
\begin{aligned}
l_{x} & =b \cdot s^{x} \cdot g^{c^{x}} \quad \text { with } \\
s & =0.99949255 \\
g & =0.99959845 \\
c & =1.10291509 \\
b & =1000401.71
\end{aligned}
$$

which leads to

$$
\begin{aligned}
\int_{\tau_{i}}^{\tau_{i}+\Delta \tau} \pi_{x}(u) d u= & \frac{l_{x+\tau_{i}}-l_{x+\tau_{i}+\Delta \tau}}{l_{x}} \\
\hat{=} & \text { the probability that a life-aged- } x \text { will survive } \tau_{i} \text { years and die within } \\
& \text { the following } \Delta \tau \text { years. }
\end{aligned}
$$

We consider the contract situation of an investor at the age of 30 years at the inception of the contract. Instead of the Wang transformation we use the age adjustment, i.e. we increase the present age of the investor by 15 years. Which implies $x=45$ in our simulation. Since the death probability of a life aged 30 is less than of a life aged 45 , this results in an increase in the probability of early termination of the contract. In addition to the pension situation we also consider the case of the investment contract. In this case, by assumption the contract runs until the contract maturity with probability 1 . The comparison of the results for the pension and investment situation allows us to discuss the impact of the possibility of early termination.

For the computation of the numerical results it is necessary to integrate the expected discounted payoffs with respect to the termination distribution. To simplify this problem we assume that the payments can only take place at the end of each investment period. This implies that early termination of the contract can only occur at time $\tau \in \underline{\underline{T}}=\left\{t_{0}<\cdots<t_{n-1}\right\}$ and the contract maturity is equal to $T=t_{N}$. The maturity of the pension and investment contracts varies in yearly steps up to 15 years with a periodic payment every second month. In case of the 15 -year contract this involves the computation of $90=15 \cdot 6$ average return put options to calculate the value of the investment fraction for a fixed interest rate guarantee. To further simplify the computation we 


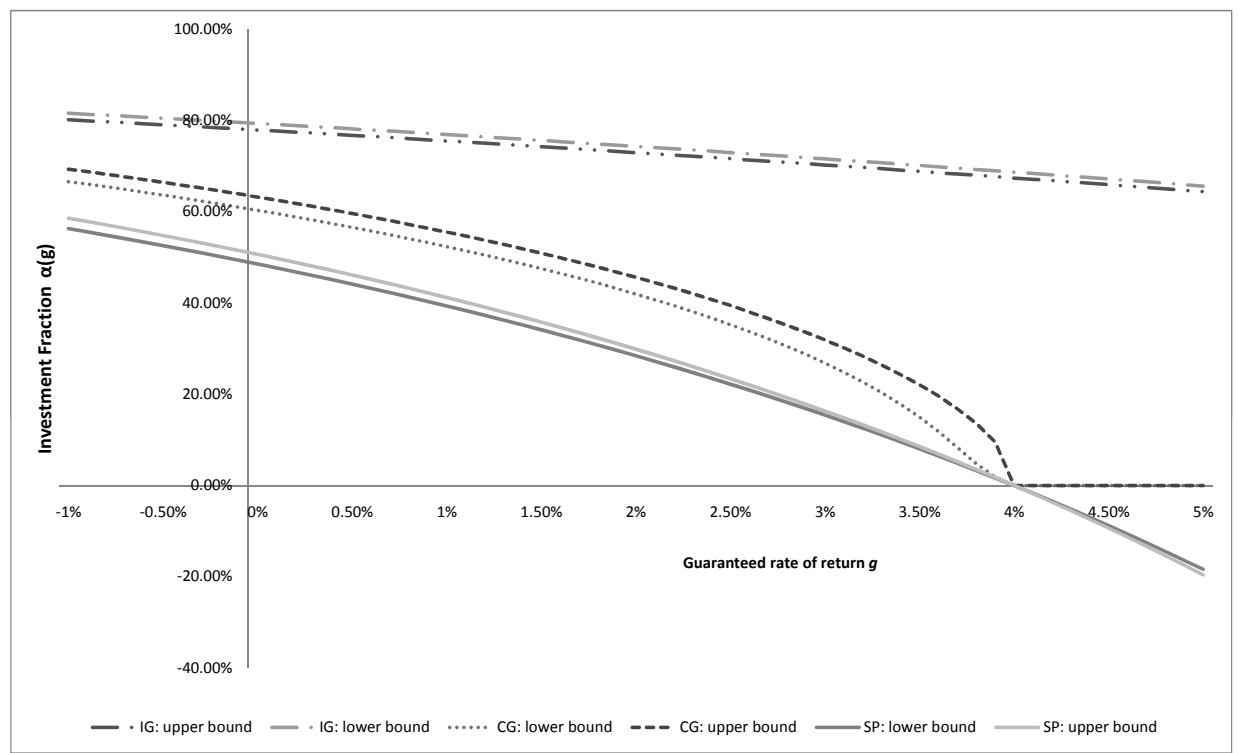

Figure 6.1: Upper and lower bound for the investment fraction $\alpha(g)$ as a function of the guaranteed rate of return $g$ for an admissible pension scheme $c \in\{I G, C G, S P\}$, contract maturity $T=15$ years, age $x=30+(15)$ and bimonthly constant contribution.

assume a constant contribution of 100 at each investment date. In case of a constant contribution the set of admissible contracts is independent of the the size of the contribution. In this particular case the corresponding equations (see (4.10), (4.11), and (4.12)) are homogenous of degree one in the constant contribution.

The central computation problem is to approximate the sequence of average return put options. Setting the maturity of the contracts to 15 years and assuming bimonthly contributions the total number of Asian-type options is 90. Since no closed form solution is available, we use the upper and lower bounds derived by the conditioning method. In addition we have to solve a second fixed point problem in the case of the contribution guaranteed scheme ( $C G$-scheme). The results are given in Figure 6.1 and Table 6.1. As a first conclusion, the approximations are quite well-behaved. Due to the second approximation problem the differences between upper and lower bounds in case of the $C G$-scheme are higher than for the other schemes. In particular for relatively high guaranteed rates of return $g$ the difference increases. In this case the solution involves the approximation of out-ofthe-money options. Although the absolute value of these options is small, the difference between the upper and lower bound is large. This finding is in line with the results on the approximation methods for Asian options as reported in Nielsen and Sandmann (2003). The behavior of the average return option is similar to the one of a standard Asian-type option.

As expected, the investment fraction $\alpha(g)$ as a function of the guaranteed rate of return is decreasing and in case of the $C G$ - and $S P$-scheme concave. The functional form of the function in case of the $I G$ - scheme is not concave, since for $g \rightarrow \infty$ the investment fraction converges to zero from above. Furthermore the graph is in line with the relative ranges of these investment fractions with respect to the different schemes, i.e. the result is consistent with Proposition 4.6. For simplicity the initial term structure was assumed to be flat with a $4 \%$ yield. Therefore in case of a guaranteed rate equal to $4 \%$ the investment share of the $C G$ - and the $S P$-scheme must be equal to zero. For higher guaranteed rates the only admissible contract of the $C G$-scheme is defined by a zero contribution sequence, whereas the $S P$-scheme implies a negative investment fraction in this case.

As reported in Table 6.1, the value of the investment fraction differs substantially across the different schemes. The $I G$-scheme results in an investment fraction of around $78 \%$ if the guaranteed rate 


\begin{tabular}{c|c|c||c|c||c|c} 
guaranteed & \multicolumn{2}{|c||}{ pension scheme $I G$} & \multicolumn{2}{c||}{ pension scheme $C G$} & \multicolumn{2}{c}{ pension scheme $P S$} \\
rate of & lower & upper & lower & upper & lower & upper \\
return $g$ & $\alpha_{I G}^{l o w}(g)$ & $\alpha_{I G}^{u p}(g)$ & $\alpha_{C G}^{l o w}(g)$ & $\alpha_{C G}^{u p}(g)$ & $\alpha_{S P}^{l o w}(g)$ & $\alpha_{P S}^{u p}(g)$ \\
\hline$-1.0 \%$ & $80.15 \%$ & $81.59 \%$ & $66.61 \%$ & $69.34 \%$ & $56.28 \%$ & $58.56 \%$ \\
$-0.5 \%$ & $79.05 \%$ & $80.49 \%$ & $63.62 \%$ & $66.44 \%$ & $52.57 \%$ & $54.78 \%$ \\
$0.0 \%$ & $77.92 \%$ & $79.34 \%$ & $60.30 \%$ & $63.21 \%$ & $48.53 \%$ & $50.66 \%$ \\
$0.5 \%$ & $76.74 \%$ & $78.16 \%$ & $56.57 \%$ & $59.60 \%$ & $44.13 \%$ & $46.14 \%$ \\
$1.0 \%$ & $75.52 \%$ & $76.92 \%$ & $52.36 \%$ & $55.54 \%$ & $39.34 \%$ & $41.20 \%$ \\
$1.5 \%$ & $74.26 \%$ & $75.65 \%$ & $47.55 \%$ & $50.93 \%$ & $34.12 \%$ & $35.80 \%$ \\
$2.0 \%$ & $72.96 \%$ & $74.33 \%$ & $41.95 \%$ & $45.63 \%$ & $28.43 \%$ & $29.88 \%$ \\
$2.5 \%$ & $71.62 \%$ & $72.97 \%$ & $35.25 \%$ & $39.43 \%$ & $22.22 \%$ & $23.40 \%$ \\
$3.0 \%$ & $70.25 \%$ & $71.57 \%$ & $26.82 \%$ & $31.90 \%$ & $15.45 \%$ & $16.31 \%$
\end{tabular}

Table 6.1: Upper and lower bound for the investment fraction $\alpha(g)$ as a function of the guaranteed rate of return $g$ for an admissible pension scheme $c \in\{I G, C G, S P\}$, contract maturity $T=15$ years, age $x=30+(15)$ and bimonthly constant contribution.

of return is assumed to be $0 \%$. For the same guaranteed rate the $C G$-scheme results in around $62 \%$ and the $S P$-scheme around $50 \%$. To understand these differences, it is necessary to consider the associated payoffs more closely. The total benefit of the different pension schemes can be decomposed into two parts. The first part consists of the guaranteed amount the investor receives at the termination of the contract. This amount is determined by the guaranteed rate of return $g$, the termination date $\tau$ and in the case of the investment guaranteed scheme $(I G)$ by the investment fraction $\alpha_{I G}(g)$. The second part is random and depends on the value of the investment portfolio at the termination date. Therefore we call this the expected surplus benefit of the contract, as opposed to the guaranteed benefit. Consider a fixed time $t_{n} \in \underline{T} \cup\left\{t_{N}\right\}$ and express these quantities in terms of money at time $t_{n}$. With respect to the definition of the three different pension schemes, the guaranteed benefit $G B_{c}$ and the expected surplus benefit $E s B_{c}$ at time $t_{n}$ are defined by

$$
\begin{aligned}
G B_{I G}\left(t_{n}, g\right) & :=\alpha_{I G}(g) \cdot A\left(t_{n}, g\right), \\
E s B_{I G}\left(t_{n}, g\right) & :=\alpha_{I G}(g) \cdot E_{Q^{t_{n}}}\left[\left[P\left(t_{n}, K\right)-A\left(t_{n}, g\right)\right]^{+}\right], \\
G B_{C G}\left(t_{n}, g\right) & :=A\left(t_{n}, g\right), \\
E s B_{C G}\left(t_{n}, g\right) & :=E_{Q^{t_{n}}}\left[\left[\alpha_{C G}(g) \cdot P\left(t_{n}, K\right)-A\left(t_{n}, g\right)\right]^{+}\right], \\
G B_{S P}\left(t_{n}, g\right) & :=A\left(t_{n}, g\right), \\
E s B_{S P}\left(t_{n}, g\right) & :=\alpha_{S P}(g) \cdot E_{Q^{t_{n}}}\left[\left[P\left(t_{n}, K\right)-A\left(t_{n}, g\right)\right]^{+}\right],
\end{aligned}
$$

where $\left(\alpha_{c}, g\right) \in I_{x, c}\left(K\left(t_{0}, \cdot, K\left(t_{N-1}\right)\right.\right.$ refers to an admissible contract with maturity $t_{N}$. Furthermore $A\left(t_{n}, g\right)$ is defined by equation (3.2) and the expected surplus part is determined as the forward value of the corresponding average rate option payoff. The difference between $t_{n}$ and $t_{N}$ can be expressed as follows. At time $t_{0}$ we consider an admissible contract $\left(\alpha_{c}, g\right)$ with contract maturity $t_{N}$. For $t_{n}<t_{N}$ the above values represent the guaranteed benefit (payment) and expected surplus if the contract termination occurs prior to the maturity. For $t_{n}=t_{N}$ these values are equal to the guaranteed benefit and expected surplus at maturity. With respect to the above decomposition of the expected payoff, the $C G$ - and the $S P$-scheme yield the same guaranteed benefit. Furthermore, the guaranteed benefit of these schemes is always larger than the one of the $I G$-scheme. The difference between the guaranteed benefit amounts is quite large, as reported in Figure 6.2. In other words, the $I G$-pension scheme is much more risky than the other two schemes.

Since the contribution sequence for all schemes is identical, and therefore also the present value of these schemes, the size of the risky investment in the $I G$-scheme is higher than for the other two schemes. Measured in terms of the expected surplus benefit, the $I G$-scheme must have a much higher average (expected) surplus benefit. This is only a statement about the expected value. 


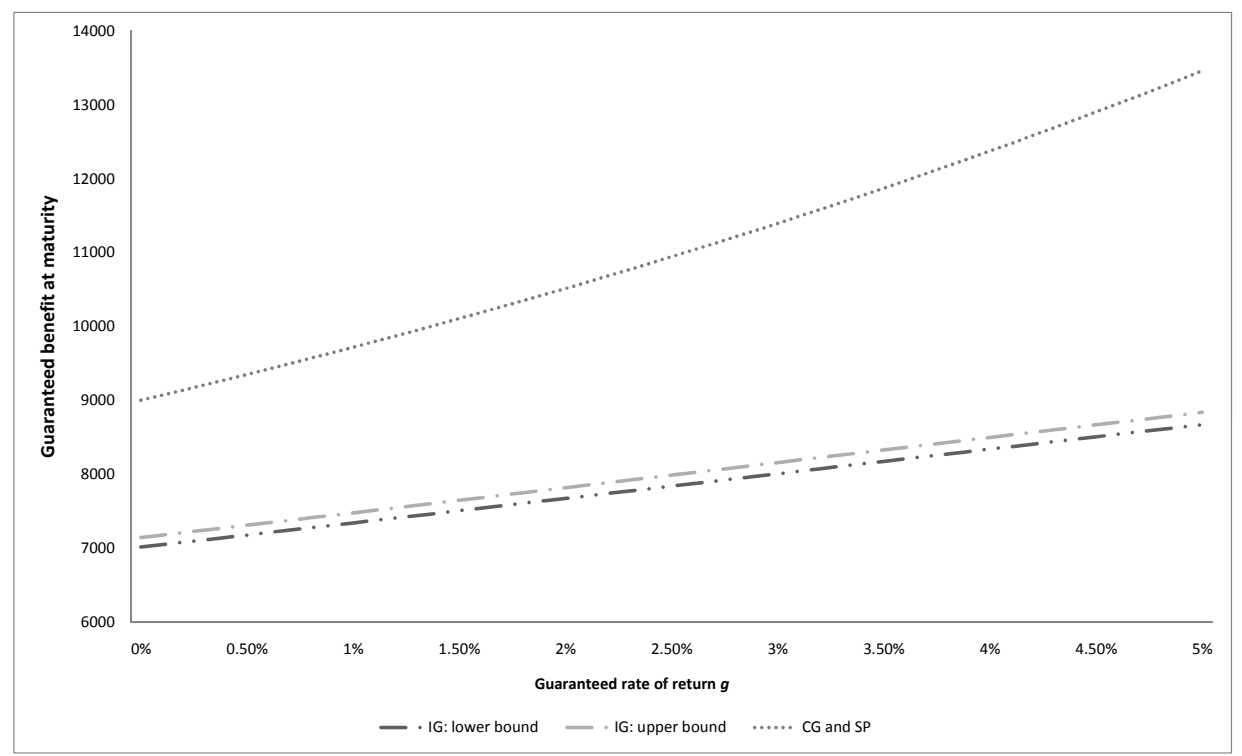

Figure 6.2: Guaranteed benefit $G B_{c}(T, g)$ at maturity as a function of the guaranteed rate of return $g$ for an admissible pension scheme $c \in\{I G, C G, S P\}$, contract maturity $T=15$ years, age $x=30+(15)$ and bimonthly constant contribution $K=100$.

Furthermore, as shown in Figure 6.3, the expected surplus benefits at maturity of the contract of the $C G$ - and $S P$-scheme are very similar. Since the present values of both contracts are identical and the guaranteed parts are identical, the expected value of the benefit must also be identical. Figure 6.4 reports this effect for the guaranteed benefit and Figure 6.5 for the expected surplus benefit, if we consider possibility of early termination. Measured in terms of the expected value we can see no difference between the $C G$ - and the $S P$-scheme. This does not mean that both schemes are equally risky. In fact the statement is only valid in terms of the expected payoffs. It would be necessary to investigate the distribution of the payoffs for the different surplus benefits on the set of admissible contracts. The conditioning method does not permit further conclusions at this point on the relative financial risk inherent in the two schemes.

The decomposition of the scheme payoffs into guaranteed benefit and expected surplus benefit also allows one to better understand the behaviour of the schemes with increasing times to maturity, as illustrated in Figure 6.6. Holding $\alpha$ and the guaranteed return $g$ constant, and for clarity of exposition assuming a flat initial term structure of interest rates, there are two opposing effects as the contract maturity is increased. As $g$ necessarily is less than the risk-free rate of interest (in the sense of Proposition 4.1), the gap between the present value of the guaranteed benefit $A\left(t_{n}\right)$ and the present value of the investor's contribution stream will widen as $t_{n}$ is increased. On the other hand, the present value of the call option payoff (the expected surplus benefit) will increase, because the present value of the "strike" $A\left(t_{n}\right)$ decreases (increasing the intrinsic value) and because the longer time to maturity results in an increase to the time value of the option. Figure 6.6 indicates that for the IG and CG schemes, the latter effect initially dominates, increasing the present value of the payoff relative to the present value of the contribution stream, and consequently the "fair" $\alpha$ must be lower for longer maturities, ceteris paribus. For the SP scheme, and at longer maturities for the IG and CG schemes, the first effect dominates, as evidenced by increasing fair $\alpha$ in contract maturity. That the first effect must be stronger in SP than IG is due to the fact that $A\left(t_{n}\right)$ is weighted less (with $\alpha<1$ ) in IG than in SP. Furthermore, writing the surplus benefit in CG as

$$
\alpha\left[P\left(t_{n}\right)-\frac{1}{\alpha} A\left(t_{n}\right)\right]^{+}
$$




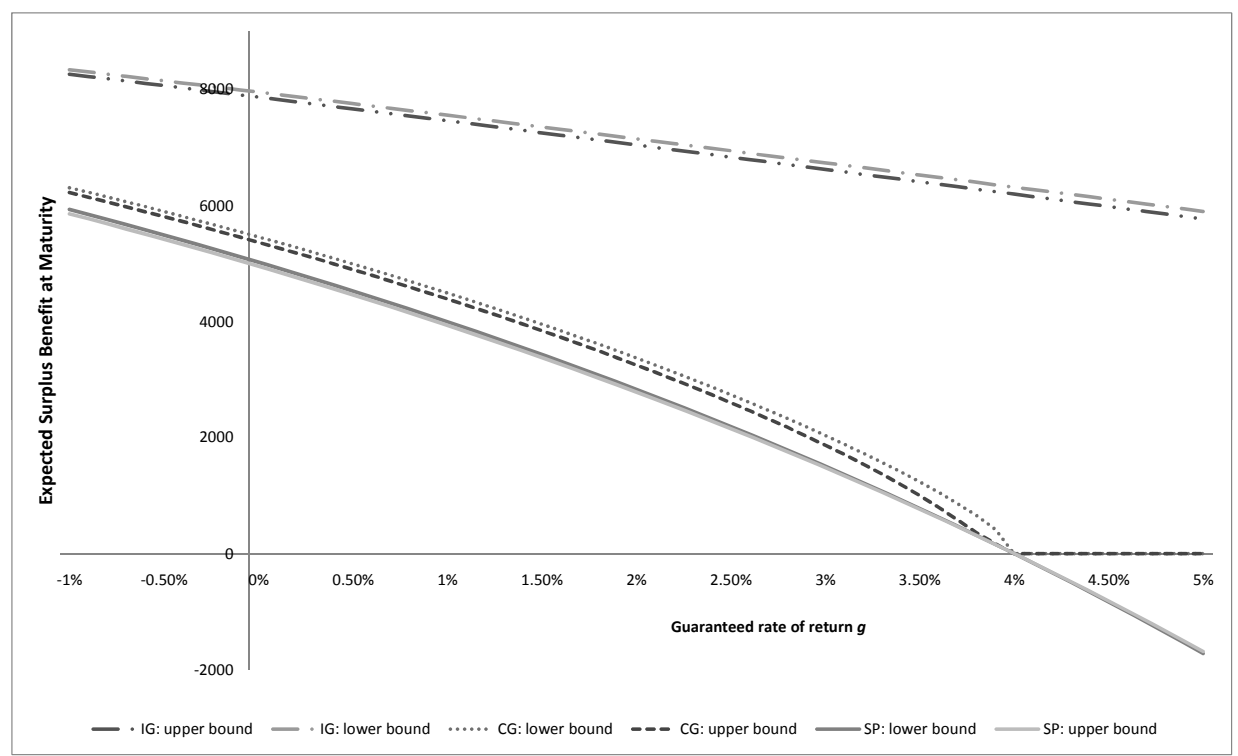

Figure 6.3: Upper and lower bounds on the expected surplus benefit at maturity $E s B_{c}(T, g)$ as a function of the guaranteed rate of return $g$ for an admissible pension scheme $c \in\{I G, C G, S P\}$, contract maturity $T=15$ years, age $x=30+(15)$ and bimonthly constant contribution $K=100$.

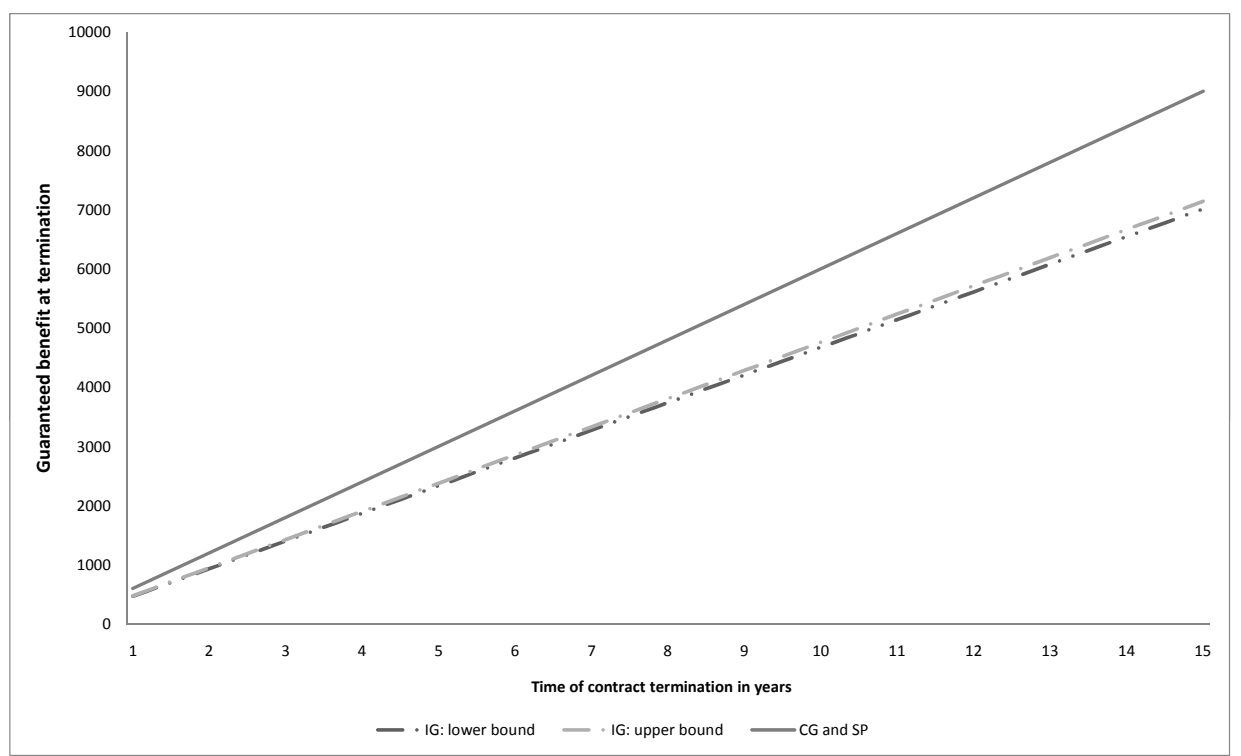

Figure 6.4: Guaranteed benefit $G B_{c}(t, g=0 \%)$ at maturity as a function of the termination date $t$ for an admissible pension scheme $c \in\{I G, C G, S P\}$, contract maturity $T=15$ years, age $x=$ $30+(15)$ and bimonthly constant contribution $K=100$. 


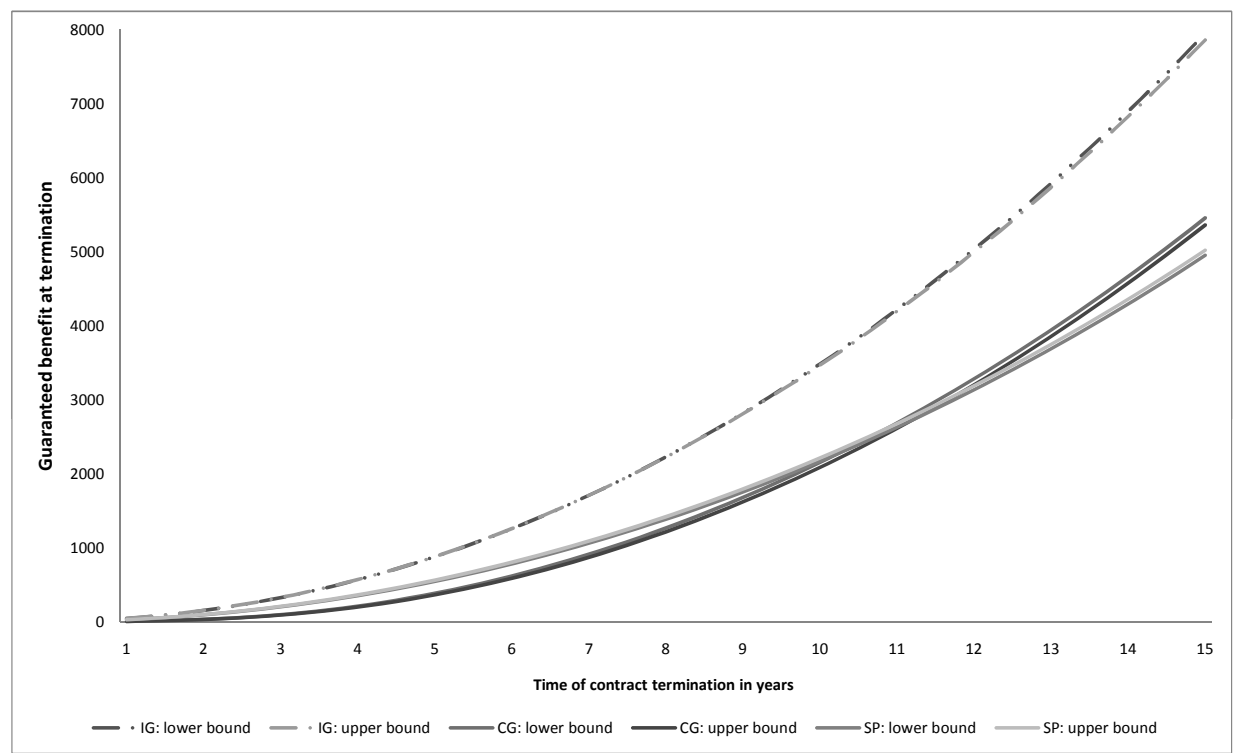

Figure 6.5: Upper and lower bounds on the expected surplus benefit at maturity $E s B_{c}(t, g=0 \%)$ as a function of the termination date $t$ for an admissible pension scheme $c \in\{I G, C G, S P\}$, contract maturity $T=15$ years, age $x=30+(15)$ and bimonthly constant contribution $K=100$.

we see that the second effect must be stronger in CG than in SP, as the intrinsic value of the option increases more quickly when $A\left(t_{n}\right)$ decreases - relative to SP, the "strike" $A\left(t_{n}\right)$ is scaled by $1 / \alpha>1$ in the CG payoff. Thus the behaviour of the schemes in increasing contract maturity as exhibited in Figure 6.6 is plausible in terms of the payoff specifications and one would expect this to hold independently of the particular choice of fixed contract and market parameters used for this illustration.

Figure 6.6 suggests that the fair investment fraction tends to a limit as the contract maturity approaches infinity. This is correct - since $g$ is less than the risk-free rate of interest, $A\left(t_{n}\right)$ decreases relative to the (forward) value of $P\left(t_{n}\right)$ as $t_{n}$ is increased, so that in forward terms the moneyness of the option increases. In the limit the call option is exercised with probability one, and thus the payoffs of the IG and CG schemes are identical. Similarly, because $g$ is less than the risk-free rate, the present value of $A\left(t_{n}\right)$ goes to zero as $t_{n}$ is increased, so for sufficiently large $t_{n}$ the present value of the payoff of all three schemes is determined by the present value of $\alpha P\left(t_{n}\right)$. In the absence of the possibility of early termination, $\alpha$ converges to 1 as $t_{n}$ goes to infinity for all three schemes (for numerical reasons, this is only evident in the upper and not in the lower bounds plotted in Figure 6.7). Allowing for early termination, payoffs at earlier maturities are weighted by the distribution of the termination date even when $t_{n}$ goes to infinity, and thus in this case the limit of $\alpha$ will be different for each scheme.

With respect to the termination distribution, the pension situation can be considered as an average over a set of investment contracts. The weights are determined by the termination distribution. Figure 6.8 to 6.10 focus on the difference between the investment and the pension situation. As an extreme case the investment situation does not allow for early termination. Apart from this difference the investment situation coincides with the corresponding pension situation. We therefore can compare two different sets of contracts. First the set of admissible pension contracts $\left(\alpha_{c}^{\text {pension }}, g\right) \in I_{x, c}^{\text {pension }}\left(K\left(t_{0}, \cdot, K\left(t_{N-1}\right)\right.\right.$ and second the corresponding set of investment contracts $\left(\alpha_{c}^{\text {invest }}, g\right) \in I_{x, c}^{\text {invest }}\left(K\left(t_{0}, \cdot, K\left(t_{N-1}\right)\right.\right.$ given the the same contribution sequence.

As shown in Figures 6.8 to 6.10, the differences between the investment proportions of admissible contract combinations as a function of the guaranteed rate of return are in all three cases very 


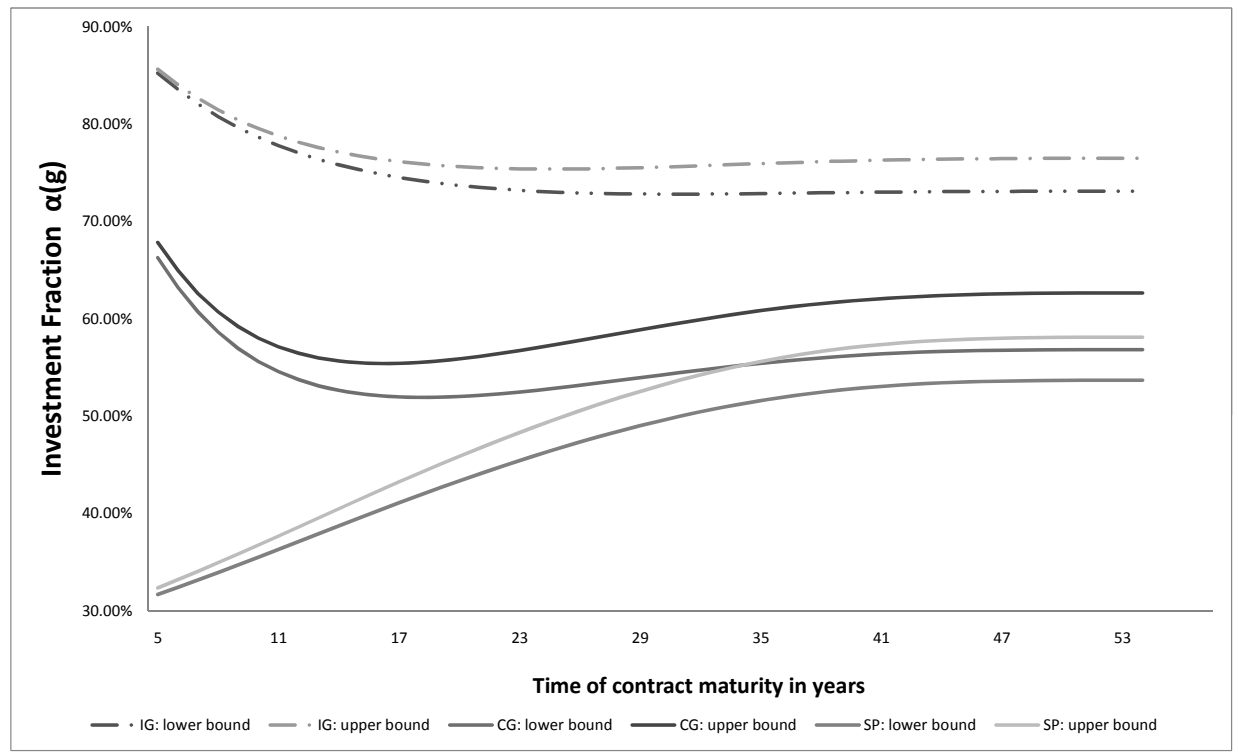

Figure 6.6: Upper and lower bound for the investment fraction $\alpha(g)$ as a function of the contract maturity for an admissible pension scheme $c \in\{I G, C G, S P\}$, guaranteed rate of return $g=1 \%$, age $x=30+(15)$ and biannual constant contribution.

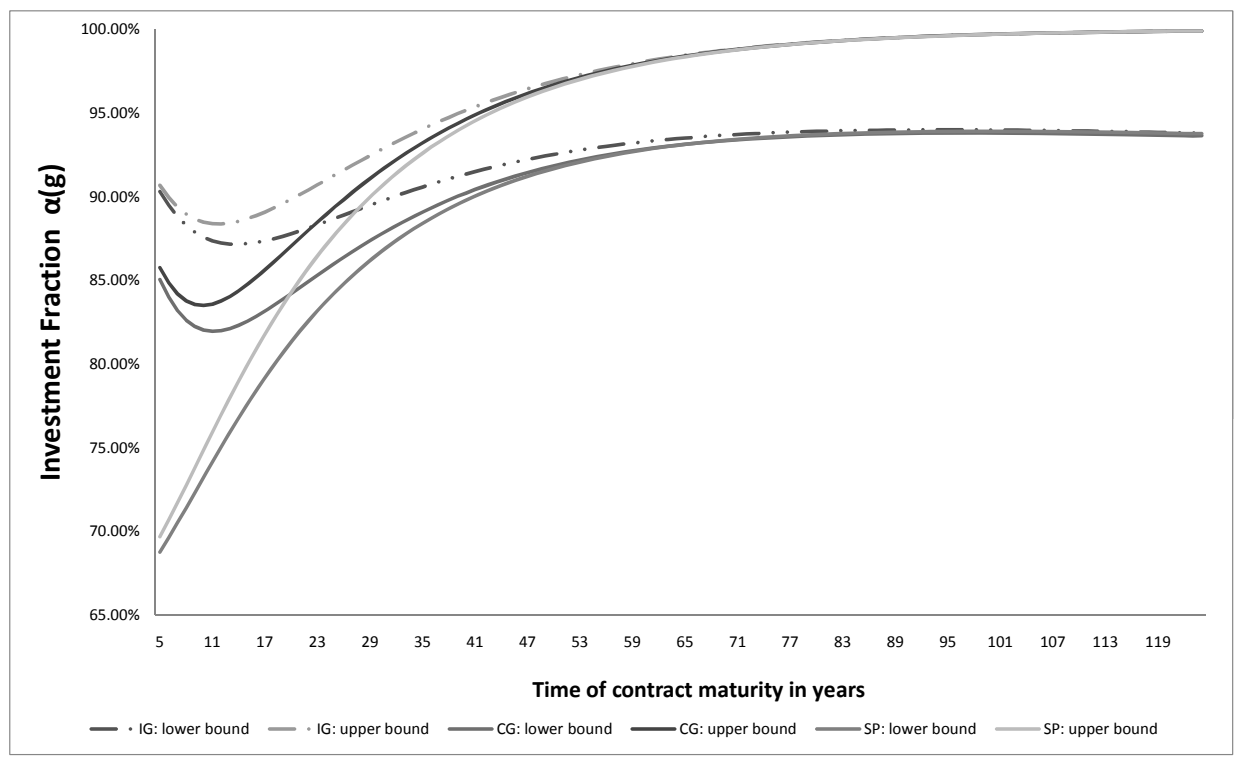

Figure 6.7: Upper and lower bound for the investment fraction $\alpha(g)$ as a function of the contract maturity for an admissible investment-only (no early termination) scheme $c \in\{I G, C G, S P\}$, guaranteed rate of return $g=-5 \%$, age $x=30+(15)$ and annual constant contribution. Low guarantee and very long contract maturities chosen to illustrate limit behaviour. 


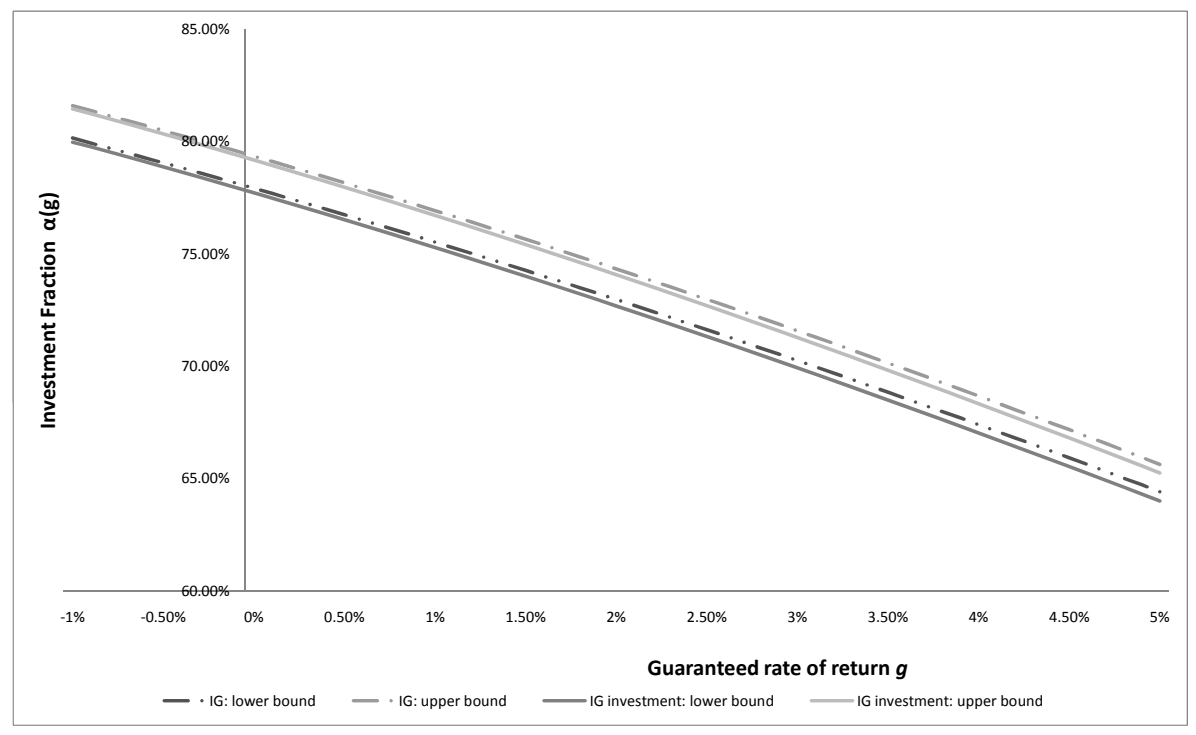

Figure 6.8: Investment Guarantee Scheme: Upper and lower bound for the investment fractions $\alpha^{\text {pension }}(g)$ and $\alpha^{\text {invest }}(g)$ as a function of the guaranteed rate of return $g$ for an admissible pension and investment schemes with contract maturity $T=15$ years, age $x=30+(15)$ and bimonthly constant contribution.

small. In fact, this illustrates the robustness of the investment proportion with respect to early termination. The termination distribution is assumed to be given by a Makeham formula. The parameter situation implies that the probability of termination prior to the maturity of the contract ( $T=15$ years) is around $12 \%$. In other words, the long term average return option payoff has a weight of at least 0.88 . Furthermore, the option values are increasing in the termination date, i.e. the contribution of the long term option dominates the options values with a shorter maturity. Both effects imply that we should expect that the investment proportion of an investment contract is no less than the corresponding investment proportion of the pension contract, but the difference can only be large if the termination distribution is much more extreme than the one considered here. These results strongly suggest that the diversification over a population is an effective risk management technique for the risk of early termination even in the presence of additional financial risk. The assumed independence between the exercise distribution and the financial risk is in this regard a worst case situation. If we assume a correlation between these risks we have the additional possibility to hedge part of the termination risk by using a trading strategy in the financial market. As in the work by Moller $(1998,2001)$ the risk minimizing strategy is thus defined by averaging the hedging strategies for each possible termination date, weighted by the termination distribution. 


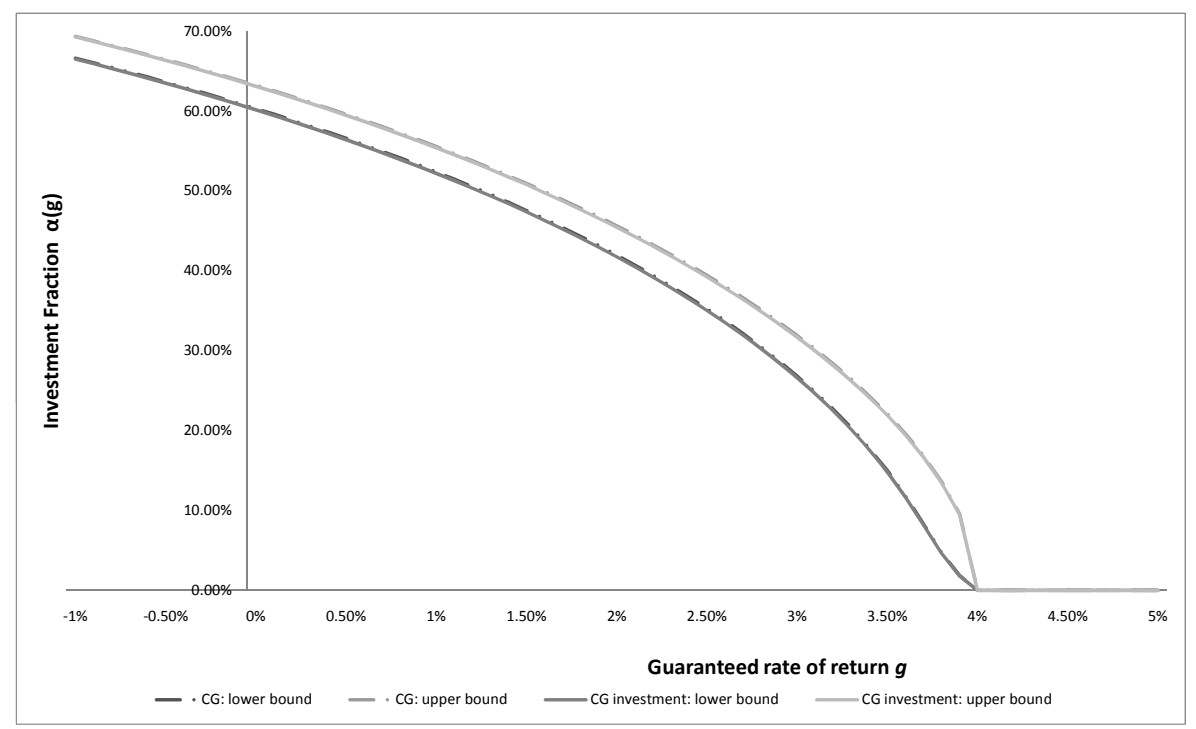

Figure 6.9: Contribution Guarantee Scheme: Upper and lower bound for the investment fractions $\alpha^{\text {pension }}(g)$ and $\alpha^{\text {invest }}(g)$ as a function of the guaranteed rate of return $g$ for admissible pension and investment schemes with contract maturity $T=15$ years, age $x=30+(15)$ and bimonthly constant contribution.

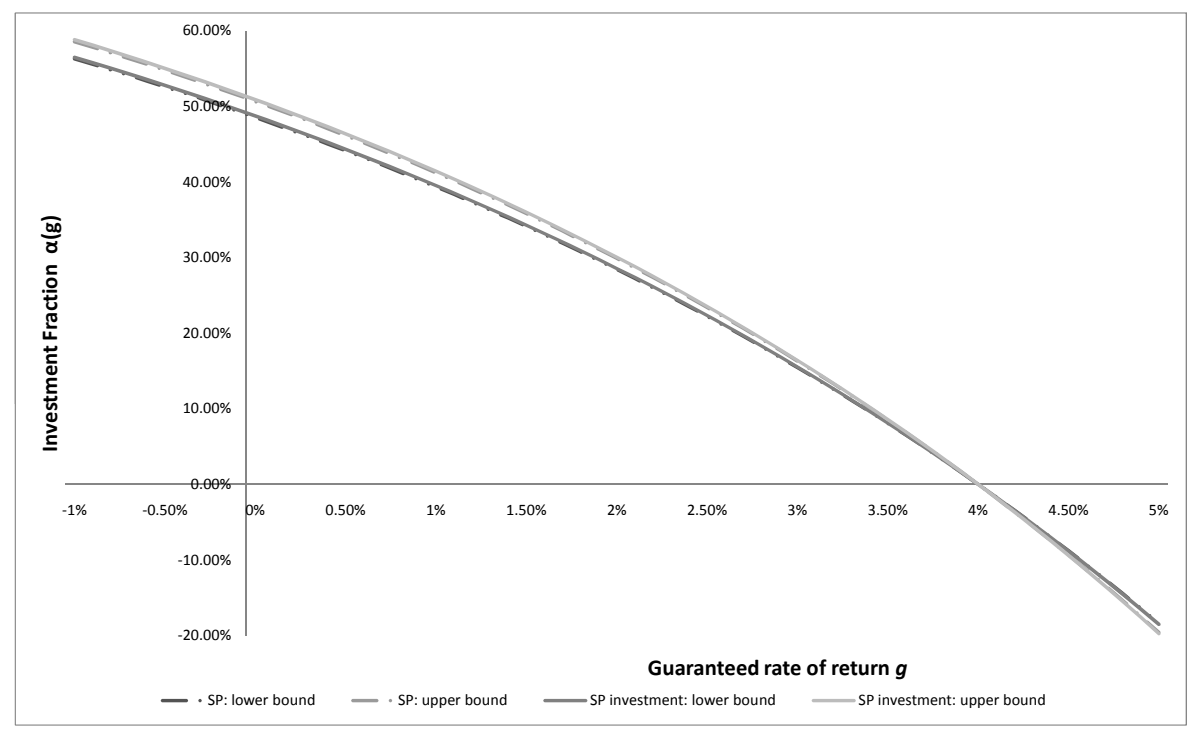

Figure 6.10: Surplus Participation Scheme: Upper and lower bound for the investment fractions $\alpha^{\text {pension }}(g)$ and $\alpha^{\text {invest }}(g)$ as a function of the guaranteed rate of return $g$ for admissible pension and investment schemes with contract maturity $T=15$ years, age $x=30+(15)$ and bimonthly constant contribution. 


\section{References}

BACINELLO, A. R. and ORTU, F. [1993]: "Pricing Equity-Linked Life Insurance with Endogenous Minimum Garantees," Insurance: Mathematics 85 Economics 12, 245-257.

BACINELlO, A. R. and ORTU, F. [1994]: "Single and Periodic Premiums for Guaranteed Equity-Linked Life Insurance under Interest-Rate Risk: The "Lognormal +Vasicek" Case," in L. PECCATI and M. VIREN (eds), Financial Modelling, Physica-Verlag, pp. 1-55.

BRENNAN, M. J. and SCHWARTZ, E. S. [1976]: "The Pricing of Equity-linked Life Insurance Policies with an Asset Value Guarantee," Journal of Financial Economics 3, 195-213.

BRENNAN, M. J. and SCHWARTZ, E. S. [1979]: "Pricing and Investment Strategies for Equitylinked Life Insurance," in L. PECCATI and M. VIREN (eds), Huebner Foundation Monograph, 7, Wharton School, University of Pennsylvania, Philadelphia.

CURRAN, M. [1994]: "Valuing Asian and Portfolio Options by Conditioning on the Geometric Mean Price," Management Science 40(12), 1705-1711.

EKERN, S. and PERSSON, S.-A. [1996]: "Exotic Unit-Linked Life Insurance Contracts," The Geneva Papers on Risk and Insurance Theory 21, 35-64.

GEMAN, H., El Karoui, N. and ROCHET, J.-C. [1995]: "Changes of Numeraire, Changes of Probability Measure and Option Pricing," Journal of Applied Probability 32, 443-458.

HEATH, D., JARROW, R. and MORTON, A. [1992]: "Bond Pricing and the Term Structure of Interest Rates: A New Methodology for Contingent Claims Valuation," Econometrica 60(1), 77-105.

MOLLER, T. [1998]: "Risk-Minimizing Hedging Strategies for Unit-Linked Life Insurance Contracts," Astin Bulletin 28, 17-47.

MOLLER, T. [2001]: "Hedging Equity-Linked Life Insurance Contracts," North American Actuarial Journal 5(2), 79-95.

NIELSEN, J. A. and SANDMANN, K. [1995]: "Equity-linked Life Insurance: A Model with Stochastic Interest Rates," Insurance, Mathematics 83 Economics 16, 225-253.

NIELSEN, J. A. and SANDMANN, K. [1996]: "Uniqueness of the Fair Premium for Equity-Linked Life Insurance Contracts," The Geneva Papers on Risk and Insurance Theory 21, 65-102.

NIELSEN, J. A. and SANDMANN, K. [2002]: "Asian Exchange Rate Options under Stochastic Interest Rates: Pricing as a Sum of Delayed Options," Finance and Stochastics 6, 355-370.

NIELSEN, J. A. and SANDMANN, K. [2003]: "Pricing Bound on Asian Options," Journal of Financial and Quantitative Analysis 38(2), 449-473.

ROGERS, L. and SHI, Z. [1995]: "The Value of an Asian Option," Journal of Applied Probability $32,1077-1088$.

VORST, T. C. [1996]: "Averaging Options," in I. NELKEN (ed.), The Handbook of Exotic Options, IRWIN Professional Publishing, Chicago, pp. 175-199. 